\title{
Modelling of Drift Wave Turbulence with a Finite Ion Temperature Gradient
}

\author{
S. Hamaguchi and W. Horton \\ Institute for Fusion Studies \\ The University of Texas at Austin \\ Austin, Texas 78712
}

$\mathrm{DOE} / \mathrm{ET} / 53088--462$

DE91 004301

\begin{abstract}
With the use of consistent orderings in $\varepsilon=\rho_{s} / a$ and $\delta=k_{\perp} \rho_{s}$ model equations are derived for the drift instabilities from the electrostatic two-fluid equations. The electrical resistivity $\eta$ included in the system allows the dynamics of both the collisional drift wave instability $(\eta \neq 0)$ and the collisionless ion temperature gradient driven instability $(\eta=0)$. The model equations used extensively in earlier nonlinear studies are obtained as appropriate limits of the model equations derived in the present work. The effects of sheared velocity flows in the equilibrium plasma and electron temperature fluctuations are also discussed.
\end{abstract}

\section{DISCLAIMER}

\begin{abstract}
This report was prepared as an account of work sponsored by an agency of the United States Government. Neither the United States Government nor any agency thereof, nor any of their employees, makes any warranty, express or implied, or assumes any legal liability or responsibility for the accuracy, completeness, or usefulness of any information, apparatus, product, or process disclosed, or represents that its use would not infringe privately owned rights. Reference herein to any specific commercial product, process, or service by trade name, trademark, inanufacturer, or otherwise does not necessarily constitute or imply its endorsement, recommendation, or favoring by the United States Government or any agency thereof. The views and opinions of authors expressed herein do not necessarily state or reflect those of the United States Government or any agency thereof.
\end{abstract}

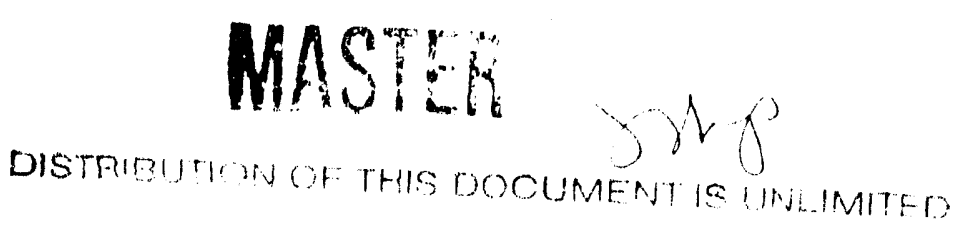




\section{Introduction}

In this work we consider the derivation of reduced nonlinear fluid equations for the description of drift wave turbulence and vortices in low beta confinement systems with magnetic shear. Numerous earlier works on drift waves contain more specialized derivations depending on a particular ordering of the several small parameters in the system. Here we generalize these earlier results in several aspects being careful to distinguish between the equilibrium expansion parameters and perturbation expansion parameters. In order to consider situations relevant to current tokamak experiments, we assume that the ambient sheared ion flows may exist in the plasma. On the other hand, for the reasons of simplicity, we neglect effects associated with trapped particles. Although kinetic effects such as those associated with ion Landau damping and trapped electrons are likely to play a prominent role in some circumstances, we only discuss the fluid model here, assuming that the fluid model approximates the dynamics of the strongly destabilized mode reasonably well, as is commonly believed.

One of the earliest accounts of the instability caused by ion temperature gradients is found in the paper by Rudakov and Sagdeev, ${ }^{1}$ where it is shown that the growth of the "ionic electrostatic" wave is caused by "a continuous inflow of heat from a region with a high unperturbed temperature into the region where the temperature is rising on account of the compression due to the plasma wave" under the conditions of zero density gradient $\left(n(x)=\right.$ const) and finite temperature gradient $\left(T_{i}(x)=T_{e}(x) \neq\right.$ const). More detailed discussions on the fluid and kinetic morlels of this instability, the dispersion relation, the critical value of $\eta_{i}$ for the marginal stability and localization of the mode are presented by Kadomtsev and Pogutse, ${ }^{2}$ based on the local approximation. Coppi, Rosenbluth, and Sagdeev ${ }^{3}$ have derived the integral equation of the instability due to ion temperature gradients from the Vlasov equation, using the normal mode analysis. The fluid limit of this kinetic model is also discussed therein. On the other hand, the model equations for instabilities of the collisional drift wave due to ion and electron temperature gradients are derived and discussed by 
Hinton and Horton ${ }^{4}$ and also by IIorton and Varma, ${ }^{5}$ based on the two-fluid equations ${ }^{6}$ with the effects of resistivity, viscosity and thermal conductivity. The ion temperature gradient driven drift instability, modelled in a slightly different way from previous model equations and called the ion-mixing mode by Coppi and Spight, ${ }^{7}$ is used to explain the rate cl density rise observed when neutral gas is fed into a tokamak plasma during a stable discharge. ${ }^{i, 8}$

Simple fluid model equations of drift waves in the absence of the ion and electron temperature gradients are derived in the collisionless limit by Hasegawa and Mima ${ }^{9}$ and in the collisional limit by Hasegawa and Wakatani. ${ }^{10,11}$ These sets of equations provides simple models of plasma turbulence, from which one can relatively easily perfurm mode-coupling analyses and study plasma-turbulence properties such as wavenumber spectra. Horton, Estes, and Biskamp ${ }^{12}$ have also presented a simple set of fluid equations of the ion temperature gradient driven turbulence in order to assess the anomalous ion thermal transport. In their model, the electron-temperature-gradient effects are excluded and only the three scalar fields of fluctuations, the electric potential $\tilde{\phi}$, the ion pressure $\tilde{p}$ and the parallel ion velocity $\tilde{v}_{\|}$, are involved. Several other simple fluid models have been proposed for the study of drift wave turbulence under various conditions. ${ }^{13}$

There are two different branches of the ion temperature gradient driven mode. One is called "slab type," which is the drift wave coupled with the ion acoustic wave that is destabilized by the local ion temperature gradient. The other is called "interchange type," 14,15 which is destabilized by bad curvature of the magnetic field lines in the presence of the finite ion temperature gradients. Since several experimental results suggested that the turbulence associated with these two branches of the ion temperature gradient driven mode were likely to be the cause of the anomalous thermal transport observed in tokamaks and stellarators, ${ }^{16}$ numerous detailed studies of the ion temperature gradient driven mode have been presented.

The goal of this work is to derive a set of reduced equations governing the slab-type ion temperature gradient driven mode, which is generalization of the model equations of Horton, 
Estes, and Biskamp. ${ }^{12}$ Although it is possible to derive the interchange-type ion-temperaturegradient-mode equations from the reduced equations (49)-(52) derived in Sec. II, we do not present the final form of the interchange-type equations in the present work. The readers who are interested in the interchange-type equations are suggested to refer to, for example, Refs. 14 and 15. When the effect of magnetic shear is stronger than the effect of magnetic field curvature, the slab-type ion temperature gradient driven instability is predicted to be excited and become a dominant source of the experimentally observed aromalous heat transport. ${ }^{17,18}$

In deriving the reduced equations, we start from the compressible two fluid equations and ignore fluctuations of magnetic field and electron temperature, as in Ref. 12. It is also assumed that the mode is localized on a particular magnetic field line (i.e., $k_{\perp} \gg k_{\|}$) and typical frequency and growth rate of the mode are much smaller than the ion cyclotron frequency. The background fields such as the mean ion temperature gradient and the ambient magnetic field vary slowly in time and space, compared to the fluctuations. The specific ordering of physical quantities of this mode is given in Subsection II.B as the $\varepsilon$-ordering. This ordering significantly simplifies the model equations as summarized in Sec. III.

\section{Basic Equations}

\section{A. Electrostatic two-fluid transport equations}

We start from the electrostatic two-fluid equations. ${ }^{6}$ For low-frequency modes with wavelengths longer than the Debye length, we may assume charge neutrality and discard the Poisson equations (the plasma approximation). Namely, we take $n_{i}=n_{e}$ and allow $\nabla \cdot \mathbf{E} \neq 0$. We also consider the case of zero-electron-mass limit (i.e., $m_{e} \rightarrow 0$ ) and the constant electron ter.perature $T_{e}$. Effects of electron temperature fluctuations will be discussed in Sec. IV. 
The set of equations then becomes

$$
\begin{aligned}
& m_{i} n_{i}\left(\frac{\partial}{\partial t}+\mathbf{v}_{i} \cdot \nabla\right) \mathbf{v}_{i}=-\nabla p_{i}+e n_{i}\left(-\nabla \Phi+\frac{\mathbf{v}_{i}}{c} \times \mathbf{B}\right)-e n_{i} \eta \mathbf{j}+\nabla \cdot \boldsymbol{\Pi}_{i} \\
& 0=-T_{e} \nabla n_{i}-e n_{i}\left(-\nabla \Phi+\frac{\mathbf{v}_{e}}{c} \times \mathbf{B}\right)+e n_{i} \eta \mathbf{j} \\
& \frac{\partial n_{i}}{\partial t}+\nabla \cdot\left(n_{i} \mathbf{v}_{i}\right)=0 \\
& \frac{\partial n_{e}}{\partial t}+\nabla \cdot\left(n_{e} \mathbf{v}_{e}\right)=0 \quad\left(n_{i}=n_{e}\right) \\
& \frac{\partial p_{i}}{\partial t}+\mathbf{v}_{i} \cdot \nabla p_{i}+\gamma p_{i} \nabla \cdot \mathbf{v}_{i}=(\gamma-1)\left(\nabla \cdot \mathbf{q}_{i}-\mathbf{I I}_{i}: \nabla \mathbf{v}_{i}\right)
\end{aligned}
$$

where

$$
\begin{aligned}
p_{i} & =n_{i} T_{i} \\
\mathrm{j} & =e n_{i}\left(\mathbf{v}_{i}-\mathrm{v}_{e}\right) \\
\mathrm{q}_{i} & =-\frac{\kappa}{\gamma-1} \nabla T_{i}+\frac{\gamma}{(\gamma-1)} \frac{n_{i} T_{i}}{\omega_{c i} m_{i}}\left(\hat{\mathrm{b}} \times \nabla T_{i}\right)
\end{aligned}
$$

and

$$
\Pi_{i}=-\nu_{\| i} \mathbf{W}^{(0)}-\nu_{\perp i}^{(1)} \mathbf{W}^{(1)}-\nu_{\perp i}^{(2)} \mathbf{W}^{(2)}-\nu_{i}^{\mathrm{FLR}} \mathbf{W}^{\mathrm{FLR}}
$$

Equations (3), (4), and (5) yield

$$
\nabla \cdot \mathrm{j}=0
$$

In the equations above, subscripts $i$ and $e$ denote the corresponding quantities of ions and electrons, respectively. The magnitude of the electron charge is denoted as $e, \omega_{c i}$ is the ion cyclotron frequency $\omega_{c i}=Z e B / m_{i} c, Z$ is the ratio of the ion charge to $e, \gamma$ is the ratio of the specific heats, $c, n_{i}, m, p, T, \Phi$, and $\mathrm{v}$ are the light velocity, the number density, mass, pressure, temperature, electrostatic potential, and velocity, respectively. The magnetic field $\mathbf{B}$ is assumed to be time-independent, satisfying $\boldsymbol{\nabla} \cdot \mathbf{B}=0$, and $\hat{\mathbf{b}}=\mathbf{B} /|\mathbf{B}|$ is the unit vector in the B-direction. Since the electrostatic limit is considered, we may ignore the Maxwell's 
equations. Therefore the current density j given by Eq. (6) anci satisfying Eq. (9) need not satisfy $\mathbf{j}=c \nabla \times \mathbf{B} / 4 \pi$. We note that the electron thermal energy balance equation is not included in the system (1)-(8) since constant electron temperature $T_{e}$ has been assumed. In the expression of the ion heat flux $\mathrm{q}_{i}$ of $\mathrm{Eq} .(\bar{T}), \boldsymbol{\kappa}$ denotes the heat conductivity tensor. The traceless tensor $\Pi_{i}$ consists of the gyroviscous tensor $\Pi_{i}^{\mathrm{FLR}}=-\nu_{i}^{\mathrm{FLR}} \mathbf{W}^{\mathrm{FLR}}$ and the collisional stress tensors $\Pi_{i}^{\text {col }}=-\nu_{\| i} \mathbf{W}^{(0)}-\nu_{\perp i}^{(1)} \mathbf{W}^{(1)}-\nu_{\perp i}^{(2)} \mathbf{W}^{(2)}$. In the Cartesian coordinates, the $(\alpha, \beta)$ component of $\Pi_{i}$ defined in Eq. $(8)$ is given ${ }^{6}$ by

$$
\begin{aligned}
& W_{\alpha \beta}^{(0)}=\frac{3}{2}\left(b_{\alpha} b_{\beta}-\frac{1}{3} \delta_{\alpha \beta}\right)\left(b_{\mu} b_{\nu}-\frac{1}{3} \delta_{\mu \nu}\right) \sigma_{\mu \nu} \\
& W_{\alpha \beta}^{(1)}=\left(\delta_{\alpha \mu}^{\perp} \delta_{\beta \mu}^{\perp}-\frac{1}{2} \delta_{\alpha \beta}^{\perp} \delta_{\mu \nu}^{\perp}\right) \sigma_{\mu \nu} \\
& W_{\alpha \beta}^{(2)}=\left(\delta_{\alpha \mu}^{\perp} b_{\beta} b_{\nu}+\delta_{\beta \nu}^{\perp} b_{\alpha} b_{\mu}\right) \sigma_{\mu \nu}
\end{aligned}
$$

and

$$
W_{\alpha \beta}^{\mathrm{FLR}}=\frac{1}{2}\left(\delta_{\alpha \mu} \varepsilon_{\beta \alpha \nu}+\delta_{\beta \nu} \varepsilon_{\alpha \gamma \mu}\right) b_{\alpha} \sigma_{\mu \nu}+\frac{3}{2}\left(b_{\alpha} b_{\mu} \varepsilon_{\beta \gamma \nu}+b_{\beta} b_{\nu} \varepsilon_{\alpha \gamma \mu}\right) b_{\alpha} \sigma_{\mu \nu}
$$

where $b_{\alpha}$ is the $\alpha$ component of $\hat{\mathrm{b}}(\alpha=1,2,3), \sigma_{\mu \nu}$ is the $(\mu, \nu)$ component of the rate-ofstrain tensor

$$
\sigma_{\mu \nu}=\partial_{\nu} v_{i \mu}+\partial_{\nu} v_{i \nu}-\frac{2}{3} \delta_{\mu \nu} \nabla \cdot \mathrm{v}_{i}
$$

$\partial_{\nu}=\partial / \partial x_{\nu}, \delta_{\mu \nu}$ is the unit tensor or the Kronecker's delta, $\delta_{\mu \nu}^{\perp}=\delta_{\mu \nu}-b_{\mu} b_{\nu}, \varepsilon_{\alpha \beta \gamma}$ is an antisymmetric unit tensor and $\nu_{i}^{\text {FLR }}=p_{i} / 2 \omega_{c i}$. In the limit of strong magnetic field $\left(\omega_{c i} \tau_{i} \gg 1\right.$, where $\tau_{i}$ is the ion-ion collision time), the viscosity coefficients are given by $\nu_{\| i}=0.96 n_{i} T_{i} \tau_{i}, \nu_{\perp i}^{(2)}=0.30 n_{i} T_{i} / \omega_{c i}^{2} \tau_{i}$ and $\nu_{\perp_{i}}^{(1)}=\nu_{\perp_{i}}^{(2)} / 4$. The divergence of the tensor in Eq. (1) is defined as $\left(\nabla \cdot \Pi_{i}\right)_{\alpha}=\partial_{\beta} \Pi_{i \alpha \beta}$ and the contraction in Eq. (5) is defined as $\Pi_{2}: \nabla v_{i}=\Pi_{i \alpha \beta} \partial_{\beta} v_{i \alpha}$. We note that $\Pi_{i}: \nabla v_{i}$ may be calculated from the formula

$$
\mathbf{W}^{(\ell)}: \nabla \mathbf{v}_{i}=\frac{1}{2} \operatorname{tr}\left(\mathbf{W}^{(\ell)}\right)^{2} \quad(\ell=1,2,3)
$$

and, in particular

$$
\mathrm{W}^{\mathrm{FLR}}: \nabla \mathrm{v}_{i}=0
$$


namely, the gyroviscosity does not produce heat.

The parallel stress tensor $\Pi_{\| i}=-\nu_{\| i} \mathbf{W}^{(0)}$ can be written in a explicit vector form as

$$
\Pi_{\| i}=-3 \nu_{\| i}\left(\hat{b} \hat{b}-\frac{1}{3} \mathrm{I}\right) \lambda,
$$

where

$$
\lambda=\hat{\mathbf{b}} \cdot\left((\hat{\mathbf{b}} \cdot \boldsymbol{\nabla}) \mathbf{v}_{i}\right)-\frac{1}{3} \boldsymbol{\nabla} \cdot \mathbf{v}_{i}
$$

and $\mathbf{I}$ is the unit tensor. U'sing Eqs. (14) and (15), we obtain

$$
\Pi_{\| i}: \nabla \mathbf{v}_{i}=-3 \lambda^{2} .
$$

The other parts of the stress tensor generally have no simple vector expressions if $\hat{b}$ is a function of space. However, if the dependence of $\hat{b}$ on the space coordinates $\mathbf{x}$ is weak in the sense that $\left|\partial_{\alpha} v_{i \beta}\right| /\left|v_{i \beta}\right| \gg\left|\partial_{\mu} b_{\nu}\right| \quad(\alpha, \beta, \mu, \nu=1,2,3)$ or, in other words, the space derivatives of the velocity $v_{i}$ are much larger than space derivatives of the unit vector $\hat{b}$, then the divergence of the stress tensor $\nabla \cdot \Pi_{i}$ in Eqs. (1) may be calculated from the following expressions:

$$
\begin{aligned}
& \boldsymbol{\nabla} \cdot \mathbf{W}^{(0)}=3 \hat{\mathbf{b}}(\hat{\mathbf{b}} \cdot \nabla) \lambda-\nabla \lambda+\mathcal{O}\left(\varepsilon^{*}\right) \\
& \nabla \cdot \mathbf{W}^{(1)}=\nabla \cdot \nabla_{\perp} \mathbf{v}_{\perp}+\mathcal{O}\left(\varepsilon^{*}\right) \\
& \nabla \cdot \mathbf{W}^{(2)}=\hat{\mathrm{b}}\left(\partial_{\|}\left(\nabla_{\perp} \cdot \mathbf{v}\right)+\nabla_{\perp} \cdot \nabla v_{\|}\right)+\partial_{\|}\left(\nabla_{\perp} v_{\|}\right)+\partial_{\|}^{2} \mathbf{v}_{\perp}+\mathcal{O}\left(\varepsilon^{*}\right) \\
& \nabla \cdot \mathbf{W}^{F} L R=-\nabla_{\perp}(\hat{\mathbf{b}} \cdot(\nabla \times \mathbf{v}))+2 \hat{\mathbf{b}} \cdot \partial_{\|}(\hat{\mathbf{b}} \cdot(\nabla \times \mathbf{v})) \\
& +\hat{\mathbf{b}} \times \nabla(\nabla \cdot \mathbf{v})+3(\hat{\mathbf{b}} \cdot \nabla)^{2}(\hat{\mathbf{b}} \times \mathbf{v})-(\hat{\mathbf{b}} \cdot \nabla)(\nabla \times \mathbf{v})+\mathcal{O}\left(\varepsilon^{*}\right) .
\end{aligned}
$$

Here $\mathcal{O}\left(\varepsilon^{*}\right)$ denotes the terms smaller than the leading terms by order of $\varepsilon^{*} \simeq$ $\left|\partial_{\mu} b_{\nu}\right| /\left(\left|\partial_{\alpha} v_{\beta}\right| /\left|v_{\beta}\right|\right)$. We have also used $\mathbf{v}_{\perp}=\delta_{\alpha \beta}^{\perp} v_{\beta}=\mathbf{v}-\hat{\mathbf{b}}(\hat{\mathbf{b}} \cdot \mathbf{v}), v_{\|}=\hat{\mathrm{b}} \cdot \mathbf{v}_{\|}$, $\nabla_{\perp}=\nabla-\hat{\mathrm{b}}(\hat{\mathrm{b}} \cdot \nabla)$ and $\partial_{\|}=\hat{\mathrm{b}} \cdot \nabla$. In Eqs. (16)-(19), the subscripts $i$ were omitted for simplicity. 
In the system of Eqs. (1)-(5), we retain the resistivity $\eta=m_{e} / n_{i} e^{2} \tau_{e}$, where $\tau_{e}\left(\tau_{e}^{-1}=\right.$ $0.51 v_{e i}^{B r}$ in terms of the collision frequency $\nu_{e i}^{B r}$ given by Braginskii ${ }^{(5)}$ is the electron collision time, as a possible nonzero coefficient while the electron mass and the electron diffusion coefficients are all set to be zero. The condition that the friction force $\mathbf{R}=n_{i} e \eta \mathbf{j}$ in Eqs. (1) and (2) has a significant effect on the dynamics is given by the following argument. In the parallel component of Eq. (2) to the magnetic field $\mathbf{B}$, balancing the first term to the last term in the right-hand side yields the relation $k_{\|} n_{i} T_{e} \sim n_{i} e \eta j_{\|} \sim\left(n_{i} e\right)^{2} \eta v_{\| i}$. We also expect from Eq. (3) that the time derivative of the density is of the same order as the parallel density flux, or $\omega n_{i} \sim k_{\|} n_{i} v_{\| i}$. Here $k_{\|}$and $\omega$ denote the parallel component of the wavenumber vector $\mathbf{k}$ to $\mathbf{B}$ and a typical frequency of the mode, respectively. Eliminating $v_{\| i}$ from these two relations, we obtain the condition for the collisional drift mode

$$
\omega \sim \frac{k_{\|}^{2} T_{e}}{m_{e} \nu_{e}},
$$

where $\nu_{e}=\tau_{e}^{-1}$ is the electron collision frequency. Since, as will be shown later, the typical frequency $\omega$ of drift waves is given by the electron diamagnetic frequency $\omega_{e}^{*}=$ $\left(T_{e} / m_{i} \omega_{c i}\right)\left(k_{y} / L_{n}\right)$ with $L_{n}$ being the ion density gradient scale length, the condition above may be written as

$$
\omega_{e}^{*} \sim \frac{k_{\|}^{2} T_{e}}{m_{e} \nu_{e}} .
$$

Since $T_{e} / m_{e}=\lambda_{\mathrm{mfp}}^{2} \nu_{e}^{2}$, where $\lambda_{\mathrm{mfp}}=\nu_{e}^{-1} \sqrt{T_{e} / m_{e}}$ is the mean free path of the electrons, the right-hand side of the condition (20) represents the inverse of the time scale of electron diffusion in the length $k_{\|}^{-1}$ along the magnetic field lines. On the other hand, the condition for the collisionless drift mode (i.e., $\eta \rightarrow 0$ in Eqs. (1) and (2)) is, given by

$$
\omega_{e}^{*} \ll \frac{k_{\|}^{2} T_{e}}{m_{e} \nu_{e}},
$$

that is, the wave motion is sufficiently slower than the electron diffusion along the magnetic field lines. These two limiting cases-the collisional drift mode and the collisionless drift mode-will be discussed in more detail later. 


\section{B. The e-ordering}

We now simplify Eqs. (1)-(9) by specifying a certain ordering of physical quantities. Since we are concerned with low-level fluctuations of the plasma governed by Eq. (1)-(9), each physical quantity may be split into two parts: the mean part, which varies slowly in time and space, and the perturbed part, which fluctuates in time and varies rapidly in space. For example, $p_{i}=p_{i 0}+\tilde{p}_{i}$, where the subscript 0 denotes the mean part and the subscript 1 denotes the fluctuating part. As typical scales, ${ }^{12}$ we choose the ion cyclotron frequency $\omega_{c i}$ to measure the frequency and the sound speed $c_{s}=\left(T_{e} / m_{i}\right)^{1 / 2}$ to measure the velocity. The lengths are then measured with the ion inertial scale length $\rho_{s}=c_{s} \omega_{c i}^{-1}$ and the electric potential is measured with $T_{e} / e$.

Since typical fusion plasmas satisfy the condition that $\rho_{s} \ll a$, where $a$ denotes a macroscopic scale length such as the minor radius of a toroidal confinement device, we use $\varepsilon=\rho_{s} / a$ as an essential small parameter in the system. Smallness of the fluctuating quantities are then assumed to be of order $\varepsilon$ or

$$
\tilde{\mathrm{v}}_{i} / c_{s} \sim \tilde{\mathrm{v}}_{e} / c_{s} \sim \tilde{n}_{i} / n_{i 0} \sim \tilde{p}_{i} / p_{i 0} \sim e \tilde{\Phi} / T_{e} \sim \mathcal{O}(\varepsilon)
$$

whereas the mean quantities are of ordei 1 , such as $p_{i 0} / m_{i} n_{i 0} T_{e} \sim \mathcal{O}(1)$. The variation of the fluctuating and mean quantities in space may be characterized by the following orderings of space derivatives: the perpendicular derivative $\nabla_{\perp}$ is of order 1 or $\rho_{s} \nabla_{\perp} \sim \mathcal{O}(1)$ and the parallel derivatives $\partial_{\|}$is of order $\varepsilon$ or $\rho_{s} \partial_{\|} \sim \mathcal{O}(\varepsilon)$ when these operators are applied to fluctuating quantities, whereas $\rho_{s} \partial_{\perp} \sim \mathcal{O}(\varepsilon)$ and $\rho_{s} \partial_{\|} \sim \mathcal{O}\left(\varepsilon^{2}\right)$ when these operators are applied to the mean quantities.

For fluctuations localized on a particular rational surface at $\psi=\psi_{s}$, where $\psi$ denotes an appropriate magnetic flux coordinate, the mean ion velocity $\mathbf{v}_{i}$ may be expanded around the rational surface as

$$
\mathbf{v}_{i 0}(\psi)=\mathbf{v}_{i 0}\left(\psi_{s}\right)+\left(\psi-\psi_{s}\right) \partial \mathbf{v}_{i 0} / \partial \psi_{s}+\cdots
$$


The second term of this expansion may be regarded as of order $\varepsilon$; i.e., $\left|\psi-\psi_{s}\right| /\left|\psi_{s}\right| \sim \mathcal{O}(\varepsilon)$ in the neighborhood of the rational surface. On the moving frame with the constant velocity $\mathrm{V}_{\mathrm{c}} \equiv \mathbf{v}_{i 0}\left(\psi \psi_{s}\right)$, the mean velocity $\mathbf{v}_{i 0}$ may be expressed as $\mathbf{v}_{i 0}=\left(\psi-\psi \psi_{s}\right) \partial \mathbf{v}_{i} / \partial \psi_{s}+\cdots$ of $\mathcal{O}(\varepsilon)$, which significantly simplifies the system of equations by eliminating terms involving $\mathrm{V}_{c}$. From now on, therefore, we always use this moving (inertial) frame to describe the system of equations. On this moving coordinate system, we may assume that $\mathrm{v}_{i 0} / c_{s} \sim \rho_{s} \nabla_{\perp} \mathrm{v}_{i 0} / c_{s} \sim$ $\mathcal{O}(\varepsilon), \mathbf{v}_{i 0}\left(\psi=\psi_{s}\right)=0, \rho_{s} \partial_{\|} \mathbf{v}_{i 0} / c_{s} \sim \mathcal{O}\left(\varepsilon^{2}\right), e \phi_{0} / T_{e} \sim \mathcal{O}(\varepsilon)$ and time derivatives $\omega_{c i}^{-1} \partial / \partial t$ of fluctuating quantities and mean quantities are of order $\varepsilon$ and $\varepsilon^{3}$, respectively.

We now consider relative sizes of the diffusion terms of Eqs. (1)-(5), assuming that the diffusion coefficients are given in the limit of high collisionality and the strong magnetic field. With the use of the natural units of the mode introduced above, the scales of the diffusion coefficients of Eqs. (7) and (8) are then given by

$$
\begin{aligned}
\eta \frac{e^{2} n_{i}}{m_{i} \omega_{c i}} & \simeq\left(\omega_{c e} \tau_{e}\right)^{-1} \\
\frac{\nu_{\| i}}{m_{i} n_{i} \rho_{s} c_{s}} & \simeq\left(\frac{T_{i}}{T_{e}}\right) \omega_{c i} \tau_{i} \\
\frac{\nu_{\perp i}^{(i)}}{m_{i} n_{i} \rho_{s} c_{s}} & \simeq\left(\frac{T_{i}}{T_{e}}\right)\left(\omega_{c i} \tau_{i}\right)^{-1} \\
\frac{\nu_{i}^{\mathrm{FLR}}}{m_{i} n_{i} \rho_{s} c_{s}} & \simeq\left(\frac{T_{i}}{T_{e}}\right) \\
\frac{\kappa_{\| i}}{n_{i} \rho_{s} c_{s}} & \simeq\left(\frac{T_{i}}{T_{e}}\right) \omega_{c i} \tau_{i} \\
\frac{\kappa_{\perp i}}{n_{i} \rho_{s} c_{s}} & \simeq\left(\frac{T_{i}}{T_{e}}\right)\left(\omega_{c i} \tau_{i}\right)^{-1} \\
\frac{n_{i} T_{i}}{\omega_{c i} m_{i}} / n_{i} \rho_{s} c_{s} & \simeq\left(\frac{T_{i}}{T_{e}}\right)
\end{aligned}
$$

where $\nu_{\| i}=n_{i} T_{i} \tau_{i}, \nu_{\perp i}^{(2)} \simeq 4 \nu_{\perp i}^{(1)} \simeq n_{i} T_{i} / \omega_{c i}^{2} \tau_{i}, \kappa_{\| i} \simeq n_{i} T_{i} \tau_{i} / m_{i}, \kappa_{\perp i} \simeq n_{i} T_{i} / m_{i} \omega_{c i}^{2} \tau_{i}$ and $\kappa \nabla=\kappa_{\perp i} \nabla_{\perp}+\kappa_{\| i} \nabla_{\|}$. From Eqs. (20) and (21), the ratio of the electron collision frequency 
$\tau_{e}^{-1}$ to the electron cyclotron frequency $\omega_{c e}=e B / m_{e} c$ satisfies

$$
\left(\omega_{c e} T_{e}\right)^{-1} \sim \mathcal{O}(\varepsilon) \quad \text { for the collisional drift mode }
$$

and

$$
\left(\omega_{c e} \tau_{e}\right)^{-1} \ll \mathcal{O}(\varepsilon) \quad \text { for the collisionless drift mode }
$$

Here we have used $k_{\|} \sim L_{n}^{-1}$ and $k_{y} L_{n} \sim \mathcal{O}(\varepsilon)$.

For the collisional drift mode, therefore, the diffusion coefficients have the following scalings under the relevant normalization specified above;

\section{Collisional drift mode}

$$
\begin{aligned}
& \eta \sim \varepsilon, \\
& \nu_{\| i} \sim \kappa_{\| i} \sim \frac{1}{\varepsilon Z^{2}}\left(\frac{m_{e}}{m_{i}}\right)^{1 / 2}\left(\frac{T_{i}}{T_{e}}\right)^{5 / 2}, \\
& \nu_{\perp i}^{(1)} \sim \kappa_{\perp i} \sim \varepsilon Z^{2}\left(\frac{m_{i}}{m_{e}}\right)^{1 / 2}\left(\frac{T_{e}}{T_{i}}\right)^{1 / 2}, \\
& \nu_{i}^{\mathrm{FLR}} \sim \frac{n_{i} T_{i}}{\omega_{c i} m_{e}} \sim \frac{T_{i}}{T_{e}} .
\end{aligned}
$$

Here we have used Eq. (29) and the relation $\left(\omega_{c i} \tau_{i}\right) /\left(\omega_{c e} \tau_{e}\right) \sim\left(m_{e} / m_{i}\right)^{1 / 2}\left(T_{i} / T_{e}\right)^{3 / 2} Z^{-2}$.

When the collisionality of the plasma is low, some expressions of the diffusion coefficients used for a collisional plasma need to be modified. In particular, the parallel diffusion coefficients $\nu_{\| i}$ and $\kappa_{\|}$should be chosen to model collisionless ion Landau effects. ${ }^{17-19}$ For the collisionless drift mode, therefore, we assume that the resistivity and all the perpendicular diffusion coefficients are given by the classical collision theory as in Eqs. (24) and (27), whereas $\nu_{\| i}$ and $\kappa_{\| i}$ are given as quantities of order 1. From Eq. (30), we have the following conditions for the collisionless drift mode: 


\section{Collisionless drift mode}

$$
\begin{aligned}
& \eta \ll \varepsilon \\
& \nu_{\perp i}^{(1)}, \nu_{\perp i}^{(2)}, \kappa_{\perp i} \ll \varepsilon Z^{2}\left(\frac{m_{i}}{m_{e}}\right)^{1 / 2}\left(\frac{T_{e}}{T_{i}}\right)^{1 / 2}, \\
& \nu_{i}^{\mathrm{FLR}} \sim \frac{n_{i} T_{i}}{u_{c i} m_{i}} \sim \frac{T_{i}}{T_{e}} .
\end{aligned}
$$

Under the scaling assumptions of magnitude of fluctuations described above, Eqs. (16)(19) may be further simplified and the following expressions of the stress tensor are obtained:

$$
\begin{aligned}
-(\nabla \cdot \Pi)_{\perp} & =\nu_{\|} \nabla_{\perp} \lambda+\nu_{\perp}^{(1)} \nabla_{\perp}^{2} \mathbf{v}_{\perp} \\
& -\nu^{\mathrm{FLR}} \nabla_{\perp} \chi-\nu^{\mathrm{FLR}}\left(\widehat{\mathrm{b}} \times \nabla(\nabla \cdot \mathbf{v})-\partial_{\|}(\nabla \times \mathrm{v})\right)+\mathcal{O}\left(\varepsilon^{3}\right), \\
-(\boldsymbol{\nabla} \cdot \boldsymbol{\Pi})_{\|} & =2 \nu_{\|} \partial_{\|} \lambda+\nu_{\perp}^{(2)} \nabla_{\perp}^{2} v_{\|}+\nu^{\mathrm{FLR}} \partial_{\|} \chi+\mathcal{O}\left(\varepsilon^{3}\right),
\end{aligned}
$$

where

$$
\begin{aligned}
& \chi=\hat{\mathrm{b}} \cdot\left(\nabla_{\perp} \times \mathrm{v}_{\perp}\right), \\
& \lambda=\partial_{\|} v_{\|}-\frac{1}{3}(\nabla \cdot \mathrm{v}),
\end{aligned}
$$

and the subscripts $i$ are omitted. As we will show shortly afterwards, the divergence of $\mathbf{v}_{i}$ is small or $\boldsymbol{\nabla} \cdot \mathbf{v}_{i} \sim \mathcal{O}\left(\varepsilon^{2}\right)$ and, therefore, $\lambda \sim \mathcal{O}\left(\varepsilon^{2}\right)$. In estimating the order of magnitude of the terms in Eqs. (31) and (32), we have used the coliisional drift mode scalings of the diffusion coefficients without taking into account the magnitude of the factor $\left(m_{e} / m_{i}\right)^{1 / 2}$. Therefore, the first term of Eq. (31) $\nu_{\| i} \nabla_{\perp} \lambda \sim \mathcal{O}(\varepsilon)$, the second term $\nu_{\perp i}^{(1)} \nabla_{\perp}^{2} \mathrm{v}_{\perp i} \sim \mathcal{O}\left(\varepsilon^{2}\right)$, the third term $\nu_{i}^{\text {FLR }} \nabla_{\perp} \chi \sim \mathcal{O}(\varepsilon)$ and the fourth term is of order $\varepsilon^{2}$. We note here that $\chi=\hat{b} \cdot\left(\nabla_{\perp} \times v_{\perp i}\right) \sim \mathcal{O}(\varepsilon)$. For Eq. (32) all the terms are of $\varepsilon^{2}$.

We now derive equations for fluctuating quantities, assuming that the mean quantities are given. We first note that taking the lowest order of the continuity equation (3) yields 
$\nabla \cdot v_{i}=C^{\prime}\left(z^{2}\right)$. Therefore. we need to determine the perpendicular ion flow velocity $\mathrm{v}_{\perp i}$ up

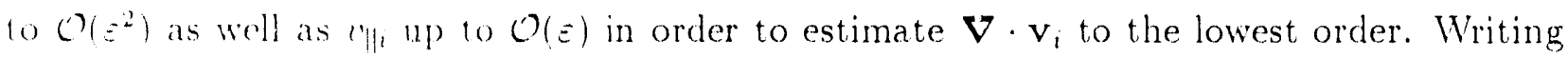
$\mathrm{v}_{\perp i}=\mathrm{v}_{\perp}^{(0)}+\mathrm{v}_{\perp}^{(1)}+\mathcal{O}\left(\sim^{3}\right)$ with $\mathrm{v}^{(0)} \sim \mathcal{O}(\varepsilon)$ and $\mathrm{v}^{(1)} \sim \mathcal{O}\left(\varepsilon^{2}\right)$, we require that $\mathrm{v}^{(0)}$ satisfy

$$
-\nabla_{\perp} p_{i}+e n_{1}\left(-\nabla_{\perp} \Phi+\mathrm{v}_{\perp}^{(0)} \times \mathrm{B},+i_{\|} \nabla_{\perp} \nabla^{(0)}-\nu_{0}^{\mathrm{FLR}} \nabla_{\perp} \hat{\mathrm{b}} \cdot\left(\nabla \times \mathrm{v}_{\perp}^{(0)}\right)=0\right.
$$

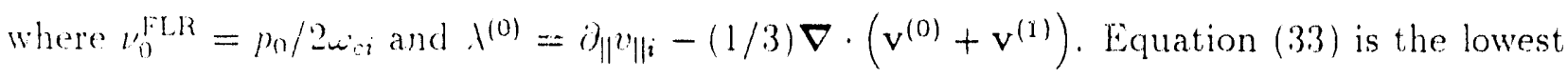
order contribution from the perpendicular components of Eq. (1). It follows from Eq. (3:3) that we may write

$$
\mathrm{v}_{\perp}^{(0)}=\mathrm{v}_{E}+\mathrm{v}_{1}+\mathrm{v}_{F}
$$

where

$$
\begin{aligned}
& \mathrm{v}_{E}=c \frac{\hat{\mathrm{b}} \times \nabla_{\perp} \Phi}{B} \\
& \mathrm{v}_{d}=c \frac{\hat{\mathrm{b}} \times \nabla_{\perp} p_{i}}{\epsilon n_{i} B}
\end{aligned}
$$

and $v_{F}$ satisfies

$$
e n_{i}\left(\frac{\mathrm{v}_{F}}{c} \times \mathrm{B}\right)=\nu_{\| i} \nabla_{\perp} \lambda^{(0)}+u_{0}^{\mathrm{FLii}} \nabla_{\perp} \hat{\mathrm{b}} \cdot\left(\nabla \times \mathrm{v}_{\perp}^{(0)}\right)
$$

In the case of the collisionless drift mode where $\nu_{\| i} \sim \mathcal{O}(1)$, the term $\nu_{\| i} \nabla_{\perp} \lambda$ should be dropped from Eqs. (33) and (37) It should be noted that $v_{\perp}^{(0)}$ contains non-fluctuating mean flows $v_{E 0}$ and $v_{d 0}$. In Appendix $A$, it is shown that the following identity $\left(E_{q} .(A-7)\right)$ holds:

$$
\frac{1}{\omega_{c i}} \hat{\mathrm{b}} \times \nabla_{\perp} p_{i} \cdot \nabla^{\prime} \|_{i i}+\left(\nabla \cdot \Pi_{i}^{\mathrm{FLR}}\right)_{\|}=p_{i} \partial_{\|}\left(\hat{\mathrm{b}} \cdot \nabla \times v_{i}\right)+\mathcal{O}\left(\varepsilon^{3}\right)
$$

The parallel component of Eq. (1) then becomes to the lowest order

$$
\begin{aligned}
& m_{i} n_{i 0}\left(\frac{\partial \tilde{v}_{\| i}}{\partial t}+\left(\mathrm{v}_{E}+\mathrm{v}_{F}\right) \cdot \nabla v_{\| i}\right)=-\partial_{\|} p_{i}-\epsilon n_{i 0} \partial_{\|} \Phi-p_{i 0} \partial_{\|}\left(\hat{\mathrm{b}} \cdot \nabla \because \mathrm{v}_{\perp}^{(0)}\right) \\
& -\epsilon n_{i 0} \eta_{j_{\|}}+2 \nu_{\| i} \partial_{\| \lambda} \lambda^{(0)}+\mu_{\perp i}^{(2)} \Delta_{\perp} \eta_{\eta_{i}} .
\end{aligned}
$$


The electron momentum equation yields

$$
\begin{aligned}
& 0=-T_{e} \nabla_{\perp} n_{i}-e n_{i}\left(-\nabla_{\perp} \phi+\frac{\mathbf{v}_{\perp} e}{c} \times \mathbf{B}\right), \\
& 0=-T_{e} \partial_{\|} n_{i}+e n_{i} \partial_{\|} \Phi+e n_{i} \eta j_{\|} .
\end{aligned}
$$

From Eqs. (3), (5), and (9), we have

$$
\begin{aligned}
& \frac{\partial \tilde{n}_{i}}{\partial t}+\mathbf{v}_{E} \cdot \nabla n_{i}+n_{i 0} \nabla \cdot \mathbf{v}_{E}+\nabla \cdot\left(n_{i} \mathbf{v}_{d}+n_{i} \mathbf{v}_{F}\right)+n_{i 0}\left(\nabla \cdot \mathbf{v}_{\perp}^{(1)}\right)+n_{i 0} \partial_{\|} v_{\| i}=0 \\
& \frac{\partial \tilde{p}_{i}}{\partial t}+\left(\mathbf{v}_{E}+\mathbf{v}_{F}\right) \cdot \nabla p_{i}+\gamma p_{i 0} \nabla \cdot\left(\mathbf{v}_{E}+\mathbf{v}_{F}\right) \\
& +\gamma p_{i 0} \nabla \cdot \mathbf{v}_{\perp}^{(1)}+\gamma p_{i 0} \partial_{\|} v_{\| i}+\gamma \nabla \cdot \frac{\hat{\mathbf{b}} \times \nabla\left(p_{i} T_{i}\right)}{m_{i} \omega_{c i}}=\nabla \cdot\left(\kappa \nabla \tilde{T}_{i}\right)
\end{aligned}
$$

and

$$
\nabla \cdot \mathbf{j}=0
$$

In deriving Eq. (43), the following identity is used:

$$
\gamma p_{i} \nabla \cdot \mathbf{v}_{d}+(\gamma-1) \nabla \cdot q^{F L R}=\gamma \nabla \cdot \frac{\hat{\mathbf{b}} \times \nabla\left(p_{i} T_{i}\right)}{m_{i} \omega_{c i}},
$$

where

$$
\mathrm{q}^{\mathrm{FLR}}=\frac{\hat{\gamma}}{\gamma-1} \cdot \frac{p_{i}}{m_{i} \omega_{c i}}\left(\hat{\mathrm{b}} \times \nabla T_{i}\right)
$$

From Eq. (40), the perpendicular components of the electron flow velocity is given by

$$
\mathrm{v}_{\perp e}=\mathrm{v}_{E}-\mathrm{v}_{d e}
$$

where

$$
\mathrm{v}_{d e}=c \frac{\hat{\mathrm{b}} \times T_{\mathrm{e}} \nabla n_{\mathrm{i}}}{e n_{\mathrm{i}} B}
$$

Since $\mathrm{j}=e n_{i}\left(\mathrm{v}_{\mathrm{i}}-\mathrm{v}_{e}\right), \mathrm{Eq}$. (4t) may be written as

$$
\nabla \cdot\left(n_{i}\left(\mathrm{v}_{d}+\mathrm{v}_{d e}\right)\right)+\nabla \cdot\left(n_{i} \mathrm{v}_{F}\right)+n_{i 0} \nabla \cdot \mathrm{v}_{\perp}^{(1)}+e^{-1} \partial_{\| j} j_{\|}=0
$$


to the lowest order. This equation gives a relationship hetween $v_{\perp}^{(1)}$ and $j \|$.

The higher order correction $v_{\perp}^{(1)}$ to the velocity field may be calculated from Eq. (1). Writing down the terms of $\mathrm{Eq} .(1)$ up to $\mathcal{O}\left(\varepsilon^{2}\right)$ with the use of $v_{\perp}^{(0)}$ and Eq. $\left(32^{\prime \prime}\right)$, we obtain

$$
m_{i} n_{i}\left(\frac{\partial}{\partial t}+\mathbf{v}_{\perp}^{(0)} \cdot \nabla\right) \mathbf{v}_{\perp}^{(0)}+\frac{e n_{i}}{c} \eta \mathbf{j}_{\perp}^{(0)}+\left(\nabla \cdot \mathbf{I}_{i}\right)_{\perp}=\frac{e n_{i}}{c}\left(\mathbf{v}_{F}+\mathbf{v}_{\perp}^{(1)}\right) \times \mathbf{B},
$$

where $\mathbf{j}_{\perp}^{(0)}=e n\left(\mathbf{v}_{d}+\mathbf{v}_{d e}\right)=c \hat{\mathbf{b}} \times \nabla_{\perp}\left(p_{i}+n_{i} T_{e}\right) / B$. Applying $\nabla \cdot \frac{\hat{\mathbf{b}}}{m_{i} \omega_{c i}} \times$ to Eq. yields

$$
\begin{aligned}
& \boldsymbol{\nabla} \cdot \hat{\mathrm{b}} \times\left(\frac{n_{i}}{\omega_{c i}}\left(\frac{\partial}{\partial t}+\mathrm{v}_{\perp}^{(0)} \cdot \nabla\right)\left(\mathrm{v}_{E}+\mathrm{v}_{F}\right)+\frac{1}{m_{i} \omega_{c i}} \nabla_{\perp} \zeta+\frac{1}{m_{i} \omega_{c i}} \nabla \cdot \Pi_{i}^{\mathrm{col}}\right) \\
& =\nabla \cdot\left(n_{i} \mathbf{v}_{F}\right)+n_{i 0} \nabla \cdot \mathrm{v}_{\perp}^{(1)},
\end{aligned}
$$

where we use the following relation

$$
m_{i} n_{i}\left(\frac{\partial}{\partial t}+\mathrm{v}_{\perp}^{(0)} \cdot \nabla\right) \mathbf{v}_{d}+\left(\nabla \cdot \Pi^{\mathrm{FLR}}\right)_{\perp}=\frac{1}{\omega_{c i}} \hat{\mathrm{b}} \times \nabla\left(\frac{\partial}{\partial t} \tilde{p}_{i}+\mathrm{v}_{\perp}^{(0)} \cdot \nabla p_{i}\right)+\nabla_{\perp} \zeta
$$

with

$$
\zeta=-\frac{p_{i}}{2 \omega_{c i}} \hat{b} \cdot \nabla \times v_{\perp}^{(0)}
$$

Equation (48) is shown to hold up to $\mathcal{O}\left(\varepsilon^{2}\right)$ in Appendix A (Eq. (A-6)). Using Eq. (45) as well as the relation

$$
j_{\|}=\frac{T_{e}}{e \eta}\left(\frac{\partial_{\|} n_{i}}{n_{i 0}}-\frac{e}{T_{e}} \partial_{\|} \Phi\right)
$$

obtained from Eq. (41), we rewrite Eq. (47) as

$$
\begin{aligned}
\nabla \cdot \hat{\mathrm{b}} & \times\left(\frac{n_{i}}{\omega_{c i}}\left(\frac{\partial}{\partial t}+\mathrm{v}_{\perp}^{(0)} \cdot \nabla\right)\left(\mathrm{v}_{E}+\mathrm{v}_{F}\right)+c \frac{n_{i} \eta}{B} \mathrm{j}_{\perp}^{(0)}+\frac{1}{m_{i} \omega_{c i}} \nabla_{\perp} \zeta+\frac{1}{m_{i} \omega_{c i}} \nabla \cdot \Pi_{i}^{\mathrm{col}}\right) \\
& -\frac{1}{\omega_{c i}^{2} m_{i}} \Delta_{\perp}\left(\frac{\partial}{\partial t} \tilde{p}_{i}+\left(\mathrm{v}_{E}+\mathrm{v}_{F}\right) \cdot \nabla p_{i}\right) \\
& =-\nabla \cdot\left(n\left(\mathrm{v}_{d}+\mathrm{v}_{d e}\right)\right)+\frac{1}{e \eta}\left(\partial_{\|}^{2} \tilde{\Phi}-\frac{T_{e}}{e n_{i 0}} \partial_{\|}^{2} \tilde{n}_{i}\right) .
\end{aligned}
$$


Similarly, Eqs. (39), (42), and (43) may be written as

$$
\begin{aligned}
& m_{i} n_{i 0}\left(\frac{\partial \tilde{v}_{\| i}}{\partial t}+\left(\mathbf{v}_{E}+\mathbf{v}_{F}\right) \cdot \nabla v^{\prime} \|_{i}\right)=-\partial_{\|} p_{i}-T_{e} \partial_{\| n_{i}}-p_{i 0} \partial_{\|}\left(\hat{\mathbf{b}} \cdot \nabla \times \mathbf{v}_{\perp}^{(0)}\right) \\
& +2 \nu_{\| i} \partial_{\|} \lambda^{(0)}+\nu_{\perp i}^{(2)} \Delta_{\perp} \tilde{v}_{\| i} \\
& \frac{\partial \tilde{n}_{i}}{\partial t}+\mathbf{v}_{E} \cdot \nabla n_{i}+n_{i 0}\left(\nabla \cdot \mathbf{v}_{E}\right)-\nabla \cdot\left(n_{i} \mathbf{v}_{d e}\right)+n_{i 0} \partial_{\|} v_{\| i}+\frac{1}{e \eta}\left(\partial_{\|}^{2} \tilde{\Phi}-\frac{T_{e}}{e n_{i 0}} \partial_{\|}^{2} \tilde{n}_{i}\right)=0 \\
& \frac{\partial \tilde{p}_{i}}{\partial t}+\left(\mathbf{v}_{E}+\mathbf{v}_{F}\right) \cdot \nabla p_{i}+\gamma p_{i 0}\left(\nabla \cdot \mathbf{v}_{E}+\partial_{\|} v_{\| i}\right)-\gamma T_{i} \nabla \cdot\left(n_{i}\left(\mathbf{v}_{d}+\mathbf{v}_{d e}\right)\right)-\gamma T_{i} \mathbf{v}_{F} \cdot \nabla n_{i} \\
& +\frac{\gamma T_{i}}{e \eta}\left(\partial_{\|}^{2} \tilde{\Phi}-\frac{T_{e}}{e n_{i 0}} \partial_{\|}^{2} \tilde{n}_{i}\right)+\gamma \nabla \cdot \frac{\hat{\mathrm{b}} \times \nabla\left(p_{i} T_{i}\right)}{m_{i} \omega_{c i}}=\nabla \cdot\left(\kappa \nabla \tilde{T}_{i}\right) .
\end{aligned}
$$

Equations (49)--(52) gives the evolution equations for the fluctuating quantities $\tilde{\Phi}, \tilde{n}_{i}, \tilde{p}_{i}$, and $\tilde{\imath}_{\| i}$.

The electric field potential $\Phi$ is uniquely determined in the following way. Dividing Eq. ( 41$)$ by $n_{i}$ and integrating the resulting equation, we obtain

$$
n_{i}=n_{i 0}(\alpha, \beta) \exp \left(\frac{e \widetilde{\Phi}}{T_{e}}+\frac{e}{T_{e}} \eta \int_{s_{0}}^{s} \tilde{j}_{\|} d s^{\prime}\right),
$$

where $\alpha$ and $\beta$ denote general magnetic coordinates and $s^{\prime}$ denotes a distance along the field line $\mathbf{B}=\nabla \alpha \times \nabla \beta$. The integration constant is chosen in such a way that $n_{i 0}(\alpha, \beta)$ represents the mean number density. In the case where $e \widetilde{\Phi} / T_{e}, e \eta \int j_{\|} d / T_{e} \ll 1$, we obtain

$$
\frac{\tilde{n}_{i}}{n_{i 0}}=\frac{e \tilde{\Phi}}{T_{e}}+\frac{e \eta}{T_{e}} \int_{s_{0}}^{s} \widetilde{j}_{\|} d s^{\prime}
$$

In order to determine $\Phi$ uniquely, therefore, we may require that

$$
\frac{\tilde{n}_{i}}{n_{i 0}}=\frac{e \tilde{\Phi}}{T_{e}}
$$

at some plane $s^{\prime}=s_{0}$ (e.g., $s_{0}=-\infty$ in the case of infinite domain). 


\section{Long-wavelength approximation}

In this section we introduce a subsidiary ordering, assuming that wavelengths of the modes are much ionger than $\rho_{s}$, that is, $k_{\perp} \rho_{s} \ll 1$. More precisely, using a small parameter $\delta$ satisfying $\varepsilon \ll \delta \ll 1$, we assume that $\rho_{s} \nabla_{\perp}=\mathcal{O}(\delta), \rho_{s} \nabla_{\|}=\mathcal{O}\left(\delta^{2} \Xi\right)$ and $\left.\omega_{c i}^{-1} j\right) / \partial t=$ $\mathcal{O}\left(\delta^{2} \varepsilon\right)$ when these operators are applied to fluctuating quantities and $\rho_{s} \nabla_{\perp}=\mathcal{O}(\delta \varepsilon)$ and $\rho_{s} \nabla_{\|}=\mathcal{O}\left(\delta^{2} \varepsilon^{2}\right)$ when these operators are applied to mean quantities. We also assume that $\eta \sim \mathcal{O}\left(\delta^{2} \varepsilon\right), \nu_{\perp i}^{(1)} \sim \nu_{\perp i}^{(2)} \sim \mathcal{O}(\varepsilon)$ and $\nu_{\| i} \sim \mathcal{O}\left(\varepsilon / \delta^{2}\right)$. Under these assumptions, it follows from $E_{\{}$s. (35)-(3i) that $\mathrm{v}_{E} \sim \mathrm{v}_{d} \sim \mathcal{O}(\delta \varepsilon), \lambda^{(0)} \sim \mathcal{O}\left(\delta^{2} \varepsilon^{2}\right)$, and $\mathrm{v}_{F} \sim \mathcal{O}\left(\delta^{3} \varepsilon\right)$ whereas $v_{\|} \sim \mathcal{O}(\varepsilon)$ Using the lowest order expression of $v_{\perp}^{(0)}=v_{E}+v_{d}$, we obtain $v_{\perp}^{(1)} \sim \mathcal{O}\left(\delta^{3} \varepsilon^{2}\right)$ from Eq. (46). Since $\nabla \cdot \mathbf{v}_{\perp}^{(1)} \sim \nabla \cdot v_{F} \sim \mathcal{O}\left(\delta^{4} \Sigma^{2}\right)$, it follows that

$$
\boldsymbol{\nabla} \cdot \mathbf{v}_{i}=\boldsymbol{\nabla} \cdot\left(\mathbf{v}_{E}+\mathbf{v}_{d}\right)+\partial_{\|} v_{\| i} \sim \mathcal{O}\left(\delta^{2} \varepsilon^{2}\right)
$$

to the lowest order. Therefore the terms $\nabla \cdot \mathbf{v}_{\perp}^{(1)}$ and $\boldsymbol{\nabla} \cdot \mathbf{v}_{F}$ in Eqs. (42) and (43) are neglected under the subsidiary orderings in this section. Taking the lowest order contributions from Eqs. (49), (50), (42), and (43), we obtain

$$
\begin{gathered}
\left(\partial_{\|}^{2} \tilde{\Phi}-\frac{T_{e}}{e n_{i 0}} \partial_{\|}^{2} \tilde{n}_{i}\right)=e \eta \nabla \cdot\left(n_{i}\left(\mathbf{v}_{d}+\mathbf{v}_{d e}\right)\right) \\
m_{i} n_{i 0}\left(\frac{\partial \tilde{v}_{\| i}}{\partial t}+\mathbf{v}_{E} \cdot \nabla v_{\| i}\right)=-\partial_{\| p_{i}}-T_{e} \partial_{\|} n_{i}+2 \nu_{\| i} \partial_{\|} \lambda^{(0)}+\nu_{\perp i}^{(2)} \Delta_{\perp} \tilde{v}_{\| i} \\
\frac{\partial \tilde{n}_{i}}{\partial t}+\mathbf{v}_{E} \cdot \nabla n_{i}+n_{i 0} \nabla \cdot \mathbf{v}_{E}+\nabla \cdot\left(n_{i} \mathbf{v}_{d}\right)+n_{i 0} \partial_{\|} v_{\| i}=0 \\
\frac{\partial \tilde{p}_{i}}{\partial t}+\mathbf{v}_{E} \cdot \nabla p_{i}+\gamma p_{i 0}\left(\nabla \cdot \mathbf{v}_{E}+\partial_{\| v_{\| i}}\right)+\gamma \nabla \cdot \frac{\hat{\mathbf{b}} \times \nabla\left(p_{i} T_{i}\right)}{e B}=\nabla \cdot\left(\kappa \nabla \tilde{T}_{i}\right),
\end{gathered}
$$

where

$$
\lambda^{(0)}=\frac{2}{3} \partial_{\| v_{\| i}}-\frac{1}{3} \nabla \cdot\left(\mathbf{v}_{E}+\mathbf{v}_{i}\right)
$$

\section{Cold ion approximation}

In this section we consider the case where the ion temperature is significantly smaller than the electron temperature; i.e., $\varepsilon \ll \delta=T_{i} / T_{e} \ll 1$. In this case, the ion Larmor radius $\rho_{i}:=$ 
$\left(T_{i} / m_{i}\right)^{1 / 2} / \omega_{c i}$ becomes much smaller than $\rho_{s}$, and therefore we assume that perpendicular wavenumber $k_{\perp}$ satisfies $k_{\perp} \rho_{i} \ll k_{\perp} \rho_{s} \sim \mathcal{O}(1)$. We also assume that the ion temperature gradient can be much greater than the typical gradient of a mean quantity or $a / L_{p}=$ $\mathcal{O}\left(\delta^{-1}\right)\left(L_{p}^{-1}=\partial \ell n p_{i 0} / \partial r\right)$, so that the perturbed ion pressure $\tilde{p}$ could scale as $\tilde{p} / p_{i 0}=$ $\mathcal{O}(\varepsilon / \delta) \ll 1$. In this scaling, we have $\nabla_{\perp} p_{i 0} \sim \nabla_{\perp} \tilde{p}_{i} \sim \nabla_{\perp} \tilde{T}_{i} \sim \mathcal{O}(\varepsilon), p_{i 0} \sim \nu_{i}^{\mathrm{FLR}} \sim \mathcal{O}(\delta)$, and $\zeta \sim \mathcal{O}(\delta \varepsilon)$. Although the diffusion coenincients $\nu_{\| i}, \nu_{\perp i}^{(1)}, \nu_{\perp i}^{(2)}, \kappa_{\|}$, and $\kappa_{\perp}$ have $T_{i} / T_{e}$ dependence, we ignore this dependence and assume that $\eta \sim \mathcal{O}(\varepsilon), \nu_{\|_{i}} \sim \kappa_{\| i} \sim \mathcal{O}\left(\varepsilon^{-1}\right)$ and $\nu_{\perp i}^{(1)} \sim \nu_{\perp i}^{(2)} \sim \kappa_{\perp} \sim \mathcal{O}(\varepsilon)$ as in Sec. II-B so that the terms involving the diffusion coefficients are still kept in the lowest order equations. Under these assumptions, it follows from Eqs. (34)-(37) that, $\mathbf{v}_{F}$ becomes of order $\delta \varepsilon$ whereas $\mathbf{v}_{E}$ and $\mathbf{v}_{d}$ are of order $\varepsilon$. The polarization drift velocity $\mathbf{v}_{p}=\mathbf{v}_{F}+\mathbf{v}_{\perp}^{(1)}$ is then obtain from Eqs. (46) and (48) to the lowest order as

$$
\begin{aligned}
\mathbf{v}_{p}= & \hat{\mathbf{b}} \times\left(\frac{1}{\omega_{c i}}\left(\frac{\partial}{\partial t}+\left(\mathbf{v}_{E}+\mathbf{v}_{d}\right) \cdot \nabla\right) \mathbf{v}_{E}+\frac{c \eta}{B} \mathbf{j}_{\perp}^{(0)}\right. \\
& \left.+\frac{1}{n_{i} m_{i} \omega_{c l}^{2}} \hat{\mathbf{b}} \times \nabla\left(\frac{\partial}{\partial t} \tilde{p}_{i}+\mathbf{v}_{E} \cdot \nabla p_{i}\right)+\frac{1}{e n_{i} m_{i} \omega_{c i}} \nabla \cdot \Pi_{i}^{\text {col }}\right) .
\end{aligned}
$$

Taking the lowest ord . components of Eqs. (49)-(52) we obtain

$$
\begin{aligned}
& \boldsymbol{\nabla} \cdot \hat{\mathrm{b}} \times\left(\frac{n_{i}}{\omega_{c i}}\left(\frac{\partial}{\partial t}+\left(\mathbf{v}_{E}+\mathbf{v}_{d}\right) \cdot \boldsymbol{\nabla}\right) \mathbf{v}_{E}+\frac{c n_{i} \eta}{B} \mathbf{j}_{\perp}^{(0)}+\frac{1}{m_{i} \omega_{c i}} \nabla \cdot \Pi_{i}^{\mathrm{col}}\right) \\
& +\frac{1}{m_{i} \omega_{c i}^{2}} \Delta_{\perp}\left(\gamma \nabla \cdot \frac{\hat{b} \times \nabla\left(p_{i} T_{i}\right)}{m_{i} \omega_{c i}}-\nabla \cdot\left(\kappa \nabla \tilde{T}_{i}\right)\right) \\
& =-\nabla \cdot\left(n_{i}\left(\mathbf{v}_{d}+\mathbf{v}_{d e}\right)\right)+\frac{1}{e \eta}\left(\partial_{\|}^{2} \tilde{\Phi}-\frac{T_{e}}{e n_{i 0}} \partial_{\|}^{2} \tilde{n}_{i}\right) \text {, } \\
& m_{i} n_{i 0}\left(\frac{\partial \tilde{v}_{\| i}}{\partial t}+\mathrm{v}_{E} \cdot \nabla v_{\| i}\right)=-\partial_{\|} p_{i}-T_{e} \partial_{\|} n_{i}+2 \nu_{\| i} \partial_{\|} \lambda^{(0)}+\nu_{\perp}^{(2)} \Delta_{\perp} \tilde{v}_{\| i}, \\
& \frac{\partial \tilde{n}_{i}}{\partial t}+\mathrm{v}_{E} \cdot \nabla n_{i}+n_{i 0}\left(\nabla \cdot \mathbf{v}_{E}\right)-\nabla \cdot\left(i_{i} \mathbf{v}_{d e}\right)+n_{i 0} \partial_{\|} v_{\|}+\frac{1}{e \eta}\left(\partial_{\|}^{2} \tilde{\Phi}-\frac{T_{e}}{e n_{i 0}} \partial_{\|}^{2} \tilde{n}_{i}\right)=0 \text {, }
\end{aligned}
$$




$$
\frac{\partial \tilde{p}_{i}}{\partial t}+\mathrm{v}_{E} \cdot \nabla p_{i}+\gamma \nabla \cdot \frac{\hat{\mathbf{b}} \times \nabla\left(p_{i} T_{i}\right)}{m_{i} \omega_{c i}}=\nabla \cdot\left(\kappa \nabla \ddot{T}_{i}\right),
$$

where

$$
\lambda^{(0)}=\frac{2}{3} \partial_{\| v_{\| i}}-\frac{1}{3} \nabla \cdot\left(\mathbf{v}_{E}+\mathbf{v}_{d}\right)
$$

Equation (62) has been used in deriving Eq. (59) from Eq. (49).

\section{Slab Models with Magnetic Shear}

In this section we consider a simple geometry of magnetic field and further simplify the basic equation of the ion temperature gradient driven mode obtained in the previous section. In the usual Cartesian coordinate system $(x, y, z)$ with the unit vectors $\tilde{\mathbf{x}}, \tilde{\mathbf{y}}$ and $\hat{\mathbf{z}}$, we assume that the magnetic field is given by

$$
\mathbf{B}(x)=B_{z}\left(\widehat{\mathbf{z}}+\frac{x-x_{0}}{L_{s}} \widehat{\mathbf{y}}\right)
$$

where $L_{s}$ denotes the shear scale length and the equation $x=x_{0}$ gives the magnetic surface on which the modes are considered to be localized. This sheared slab magnetic field models a local magnetic field configuration near a mode rational surface in a fusion device, such as tokamaks and reversed field pinches. In this section we are particularly concerned with a weak shear case $\left(a \ll L_{s}\right.$, where $a$ denotes a typical macroscopic length such as the minor radius of a tokamak), which is appropriate for a tokamak with small toroidal curvature. Under this weak-shear assumption, we have $B \equiv|\mathbf{B}(x)| \simeq B_{z}$ and all the terms which have the form $\nabla \cdot(\hat{\mathbf{b}} \times \nabla f)$ such as $\nabla \cdot \mathbf{v}_{E}$ and $\nabla \cdot n \mathbf{v}_{d}$ drop from the mode equations since these terms can be shown to be too small. Here we also assume that all the mean quantities are functions of only $x$ and evaluated at the mode rational surface $x=x_{0}$. In particular, we use the following space scales

$$
L_{n}=-\left(\frac{d}{d x} \ln n_{i 0}\right)^{-1} \quad, \quad L_{T}=-\left(\frac{d}{d x} \ln T_{i 0}\right)^{-1}
$$


and the nondimensional parameters

$$
\begin{aligned}
\eta_{i} & =\frac{L_{n}}{L_{T}}, \quad K=\frac{T_{i}}{T_{e}}\left(1+\eta_{i}\right), \quad \Gamma=\frac{\gamma T_{i}}{T_{e}}, \\
S_{\perp} & =\frac{L_{n} V_{0}^{\prime}}{c_{s}}, \quad S_{\|}=\frac{L_{n}}{c_{s}} \frac{d v_{\| 0}}{d x},
\end{aligned}
$$

where the perpendicular shear flow $V_{0}$ is assumed to be given by the $\mathbf{E} \times \mathbf{B}$ flow caused by the mean electric potential

$$
\Phi_{0}=\frac{1}{2 c}\left(x-x_{0}\right)^{2} B V_{0}^{\prime}
$$

with $V_{0}^{\prime}=d V_{0} / d x$ evaluated at $x=x_{0}$. The appropriate nondimensional space-time variables are

$$
\tilde{x}=\frac{x-x_{0}}{\rho_{s}}, \quad \tilde{y}=\frac{y}{\rho_{s}}, \quad \tilde{z}=\frac{z}{L_{n}}, \quad \tilde{t}=\frac{t c_{s}}{L_{n}}
$$

and the nondimensional dependent variables are

$$
\begin{array}{ll}
\phi=\frac{e \tilde{\Phi}}{T_{e}} \frac{L_{n}}{\rho_{s}}, & n=\frac{\tilde{n}_{i}}{n_{i 0}} \frac{L_{n}}{\rho_{s}}, \\
v=\frac{\tilde{v}_{\| i}}{c_{s}} \frac{L_{n}}{\rho_{s}}, & p=\frac{\tilde{p}_{i} T_{i}}{p_{i 0} T_{e}} \frac{L_{n}}{\rho_{s}} .
\end{array}
$$

With the use of the nondimensional variables defined above, Eqs. (54)-(58) of the longwavelength drift waves may be further simplified in the slab geometry. Equation (54), which becomes $\partial_{\|}^{2}(\phi-n)=0$ in the slab geometry, and the condition given in Eq. (53) yields

$$
\phi=n .
$$

Using this relation, we derive from Eqs. (55)-(58)

$$
\begin{aligned}
& \frac{\partial \phi}{\partial \tilde{t}}=-\left(1+S_{\perp} \tilde{x}\right) \frac{\partial \phi}{\partial \tilde{y}}-\tilde{\partial}_{\|} v \\
& \frac{\partial v}{\partial \widetilde{t}}+\{\dot{\phi}, v\}=-S_{\perp} \tilde{x} \frac{\partial v}{\partial \tilde{y}}+S_{\|} \frac{\partial \phi}{\partial \tilde{y}}-\tilde{\partial}_{\|}(p+n)+\mu_{\perp}^{(2)} \widetilde{\Delta}_{\perp} v+\mu_{\|} \tilde{\partial}_{\|} \lambda^{(0)} \\
& \frac{\partial p}{\partial \tilde{t}}+\{\phi, p\}=-S_{\perp} \tilde{x} \frac{\partial p}{\partial \tilde{y}}-K \frac{\partial \phi}{\partial \tilde{y}}-\Gamma \tilde{\partial}_{\|} v+\chi_{\perp} \tilde{\Delta}_{\perp} p+\chi_{\|} \tilde{\partial}_{\|}^{2} p
\end{aligned}
$$


where

$$
\begin{aligned}
\tilde{\partial}_{\|} & =s \tilde{x} \frac{\partial}{\partial \tilde{y}}+\frac{\partial}{\partial \tilde{z}}, \\
\widetilde{\nabla}_{\perp} & =\widehat{\mathbf{x}} \frac{\partial}{\partial \tilde{x}}+\widehat{y} \frac{\partial}{\partial \tilde{y}}, \quad \widetilde{\Delta}_{\perp}=\widetilde{\nabla}_{\perp}^{2}
\end{aligned}
$$

and

$$
\{f, g\}=\frac{\partial f}{\partial \widetilde{x}} \frac{\partial g}{\partial \widetilde{y}}-\frac{\partial f}{\partial \widetilde{y}} \frac{\partial g}{\partial \widetilde{x}} .
$$

In deriving Eq. (66), the term $\nabla \cdot\left(\kappa \nabla \tilde{T}_{i}\right)$ of Eq. (C-4) is replaced by $\nabla \cdot\left(n_{0}^{-1} \kappa \nabla \tilde{p}_{i}\right)$ for simplicity. The normalized diffusion coefficients are given by

$$
\begin{array}{cc}
\mu_{\|}=\frac{4 \nu_{\| i}}{3 m_{i} n_{i 0} c_{s} L_{n}}, \quad \chi_{\|}=\frac{\kappa_{\|}}{n_{i 0} c_{s} L_{n}} \\
\mu_{\perp}^{(2)}=\frac{\nu_{\perp i}^{(2)} L_{n}}{m_{i} n_{i 0} c_{s} \rho_{s}^{2}}, \quad \chi_{\perp}=\frac{\kappa_{\perp} L_{n}}{n_{i 0} c_{s} \rho_{s}^{2}} .
\end{array}
$$

In the case of the low-ion temperature drift waves, we obtain the following nondimensional form of Eqs. (59)-(62):

$$
\begin{aligned}
& \frac{\partial}{\partial \tilde{t}} \tilde{\Delta}_{\perp} \phi+\left\{\phi, \tilde{\Delta}_{\perp} \phi\right\}=\left(K-S_{\perp} \tilde{x}\right) \frac{\partial}{\partial \tilde{y}} \tilde{\Delta}_{\perp} \phi+S_{\perp} \frac{\partial^{2} p}{\partial \tilde{x} \partial \tilde{y}}+\left\{\tilde{\Delta}_{\perp} \phi, p\right\} \\
&+\left\{\frac{\partial \phi}{\partial \tilde{x}}, \frac{\partial p}{\partial \tilde{x}}\right\}+\left\{\frac{\partial \phi}{\partial \tilde{y}}, \frac{\partial p}{\partial \tilde{y}}\right\}-\frac{1}{\sigma_{\|}} \tilde{\Delta}_{\perp}(p+n)-\tilde{\Delta}_{\perp}\left(\chi_{\perp} \tilde{\Delta}_{\perp} p+\chi_{\|} \tilde{\partial}_{\|}^{2} p\right) \\
&-\dot{\sigma}_{\|} \tilde{\partial}_{\|}^{2}(\phi-n)+\mu_{\perp}^{(1)} \tilde{\Delta}_{\perp}^{2}(\phi+p) \\
& \frac{\partial n}{\partial \tilde{t}}+\{\phi, n\}=-S_{\perp} \tilde{x} \frac{\partial n}{\partial \tilde{y}}-\frac{\partial \phi}{\partial \tilde{y}}-\tilde{\partial}_{\|} v-\sigma_{\|} \tilde{\partial}_{\|}^{2}(\phi-n) \\
& \frac{\partial v}{\partial \tilde{t}}+\{\phi, v\}=-S_{\perp} \tilde{x} \frac{\partial v}{\partial \tilde{y}}+S_{\|} \frac{\partial \phi}{\partial \tilde{y}}-\partial_{\|}(p+n)+\mu_{\perp}^{(2)} \tilde{\Delta}_{\perp} v+\mu_{\|} \tilde{\partial}_{\|} \lambda^{(0)} \\
& \frac{\partial p}{\partial \tilde{t}}+\{\phi, p\}=-S_{\perp} \tilde{x} \frac{\partial p}{\partial \tilde{y}}-K \frac{\partial \phi}{\partial \tilde{y}}+\chi_{\perp} \tilde{\Delta}_{\perp} p+\chi_{\|} \tilde{\partial}_{\|}^{2} p .
\end{aligned}
$$


Here the normalized electric conductivity $\sigma_{\|}$and perpendicular viscosity $\mu_{\perp}^{(1)}$ are given by

$$
\sigma_{\|}=\frac{m_{i} \omega_{c i} \rho_{s}}{n_{i 0} \eta e^{2} L_{n}}
$$

and

$$
\mu_{\perp}^{(1)}=\frac{\nu_{\perp}^{(1)} L_{n}}{m_{i} n_{i 0} c_{s} \rho_{s}^{2}} .
$$

We note that Eqs. (63) and (64) may be reduced from Eqs. (67) and (68), respectively, under the long-wavelength approximation, whereas Eq. (70) of the perturbed pressure $p$ is the $\Gamma \rightarrow 0$ limit of Eq. (66). The equations for the parallel velocity $v$ (Eqs. (65) and (69)) are the same in both of the approximations. Exploiting these similarities of both the sets of equations, we combine these equations and propose the following set of equations, which holds both in the long-wavelength approximation and the low-ion-temperature approximation in the sheared slab magnetic field. From now on, we will drop the tildes from the independent variables for simplicity in this section.

$$
\begin{aligned}
& \frac{\partial}{\partial t} \Delta_{\perp} \phi+\left\{\phi, \Delta_{\perp} \phi\right\}=\left(K-S_{\perp} x\right) \frac{\partial}{\partial y} \Delta_{\perp} \phi+S_{\perp} \frac{\partial^{2} p}{\partial x \partial y} \\
& +\left\{\Delta_{\perp} \phi, p\right\}+\left\{\frac{\partial \phi}{\partial x}, \frac{\partial p}{\partial x}\right\}+\left\{\frac{\partial \phi}{\partial y}, \frac{\partial p}{\partial y}\right\}+\partial_{\| j} j \mu_{\perp}^{(1)} \Delta_{\perp}^{2} \phi \\
& \frac{\partial n}{\partial t}+\{\phi, n\}=-S_{\perp} x \frac{\partial n}{\partial y}-\frac{\partial \phi}{\partial y}-\partial_{\|} v+\partial_{\|} j \\
& \frac{\partial v}{\partial t}+\{\phi, v\}=-S_{\perp} x \frac{\partial v}{\partial y}-S_{\|} \frac{\partial \phi}{\partial y}-\partial_{\|}(p+n)+\mu_{\perp}^{(2)} \Delta_{\perp} v+\mu_{\|} \partial_{\|}^{2} v \\
& \frac{\partial p}{\partial t}+\{\phi, p\}=-S_{\perp} x \frac{\partial p}{\partial y}-K \frac{\partial \phi}{\partial y}-\Gamma \partial_{\|} v+\chi_{\perp} \Delta_{\perp} p+\chi_{\|} \partial_{\|}^{2} p
\end{aligned}
$$

where the nondimensionalized parallel electric current is given by

$$
j / \sigma_{\|}=\partial_{\|}(n-\phi)
$$

In Eq. (71), the diffusion terms $\sigma_{\|}^{-1} \Delta_{\perp}(p+n), \Delta_{\perp}\left(\chi_{\perp} \Delta_{\perp} p+\chi_{\|} \partial_{\|}^{2} p\right)$ and $\mu_{\perp}^{(1)} \Delta_{\perp}^{2} p$, which are kept in Eq. (67), have been dropped for the reasons of simplicity. Although dropping these 
diffusion terms from Eq. (71) is not consistent from the point of view of the collisional-driftwave ordering discussed in Sec. II-B, it does not change the relevant dynamics of strongly destabilized modes, which tend to have smaller wavenumbers $k_{\perp}$ and $k_{\|}$. We also note that, since plasmas in most fusion devices are either collisionless or marginally collisional, the diffusion coefficients $\mu_{\perp}^{(1)}, \mu_{\perp}^{(2)}, \chi_{\perp}$, and $\sigma_{\|}^{-1}$ are small whereas the parallel diffusion coefficients $\mu_{\|}$and $\chi_{\|}$are of order unity, which model the ion Landau effects. The diffusion terms $\mu_{\perp}^{(1)} \Delta_{\perp}^{2} \phi$ in Eq. (71), $\mu_{\perp}^{(2)} \Delta_{\perp} v$ in Eq. (73) and $\chi_{\perp} \Delta_{\perp} p$ in Eq. (74) are, however, retained as the energy sinks of high $k_{\perp}$ modes in a turbulence state of the mode. In Eq. $(73), \lambda^{(0)}$ is replaced by $\partial_{\|} v_{\|}$and the term $\mu_{\|} \partial_{\|}^{2} v$, together with the term $\chi_{\|} \partial_{\|}^{2} p$ in Eq. (74), is retained as a simple model of the ion Landau effects associated with the parallel motion of the plasma. One would need to use the kinetic equations in order to study the dynamics which could be strongly affected by the diffusion, such as the dynamics of the marginally stable mode.

The domain on which Eqs. (71)-(74) are solved may be given by the cubic box $-L_{x} \leq$ $x \leq L_{x}, 0 \leq y \leq L_{y}$, and $0 \leq z \leq L_{z}$. Here $L_{y}$ and $L_{z}$ are constants of order unity (note that $x, y$, and $z$ are normalized here: $x / \rho_{s} \rightarrow x, y / \rho_{s} \rightarrow y$, and $z / L_{n} \rightarrow z$ ) whereas $L_{x}$ is taken to be large enough, so that when there is magnetic shear $\left(L_{s} \neq \infty\right)$, single helicity modes localized at $x=0$ decay sufficiently at $|x| \rightarrow L_{x}$. In the case of zero magnetic shear $2 L_{x}$ represents the width of the constant background fields. The boundary conditions of Ecss. (71)-(74) for this domain are that all the der endent variables are assumed to vanish at $x=L_{x}$ and to be periodic in the $y$ and $z$-directions.

The energy balance equation associated with the set of equations (71)-(74) is given by

$$
\frac{d}{d t} E_{t}=S_{\perp}\left\langle\frac{\partial p}{\partial x} \frac{\partial \phi}{\partial y}\right\rangle-S_{\|}\left\langle v \frac{\partial \phi}{\partial y}\right\rangle-\left\langle n \frac{\partial \phi}{\partial y}\right\rangle-\frac{K}{\Gamma}\left\langle p \frac{\partial \phi}{\partial y}\right\rangle-W_{D},
$$

where the energy $E_{t}$ of the fluctuations is given by

$$
E_{t}=\frac{1}{2}\left(\left\langle n^{2}\right\rangle+\left\langle\left|\nabla_{\perp} \phi\right|^{2}\right\rangle+\left\langle v^{2}\right\rangle+\frac{1}{\Gamma}\left\langle p^{2}\right\rangle\right)
$$


and the energy sink by

$$
\begin{aligned}
W_{D} & =\mu_{\perp}^{(1)}\left\langle\left|\Delta_{\perp} \phi\right|^{2}\right\rangle+\mu_{\perp}^{(2)}\left\langle\left|\nabla_{\perp} v\right|^{2}\right\rangle+\mu_{\|}^{(2)}\left\langle\left|\partial_{\|} v\right|^{2}\right\rangle \\
& +\frac{\chi_{\perp}}{\Gamma}\left\langle\left|\nabla_{\perp} p\right|^{2}\right\rangle+\frac{\chi_{\|}}{\Gamma}\left\langle\left|\partial_{\| p}\right|^{2}\right\rangle+\sigma_{\|}\left\langle\left|\partial_{\|}(\phi-n)\right|^{2}\right\rangle .
\end{aligned}
$$

Here \langle\rangle denotes the space average of the contained quantity over the domain or

$$
\langle\rangle=\frac{1}{2 L_{x} L_{y} L_{z}} \int_{-L_{x}}^{L_{x}} d x \int_{0}^{L_{y}} d y \int_{0}^{L_{z}} d z .
$$

If $L_{x}$ is taken to be $\infty$, the normalization factor $1 / 2 L_{x}$ needs to be chosen appropriately. ${ }^{17}$ The four transport fluxes in Eq. (76)

$$
\begin{aligned}
\left\langle\frac{\partial p}{\partial x} \frac{\partial \phi}{\partial x}\right\rangle & \propto\left\langle\tilde{v}_{d y} \tilde{v}_{E x}\right\rangle, \\
\left\langle v \frac{\partial \phi}{\partial y}\right\rangle & \propto\left\langle\tilde{v}_{\|} \tilde{v}_{E x}\right\rangle, \\
\left\langle n \frac{\partial \phi}{\partial y}\right\rangle & \propto\left\langle\tilde{n}_{i} \tilde{v}_{E x}\right\rangle,
\end{aligned}
$$

and

$$
\left\langle p \frac{\partial \phi}{\partial y}\right\rangle \times\left\langle\widetilde{p}_{i} \widetilde{v}_{E x}\right\rangle
$$

where $\tilde{v}_{E x}=-c B^{-1} \partial \tilde{\Phi} / \partial y$ and $\tilde{v}_{d y}=c\left(e n_{i} B\right)^{-1} \partial \tilde{p}_{i} / \partial x$, are proportional to the transverse transports of the $y$ component of the perturbed diamagnetic flow $v_{d y}$, the perturbed parallel flow $v_{\| i}$, thie perturbed density $\tilde{n}_{i}$ and the perturbed ion pressure $\tilde{p}_{i}$, respectively.

In the collisionless limit or $\sigma_{\|} \rightarrow \infty$, the set of Eqs. (71)-(75) may be further simplified. From Eqs. (71), (72), and (75), the adiabatic electron relation $\phi=n$ (or $\tilde{n}_{i} / n_{i 0}=e \widetilde{\Phi} / T_{e}$ ) is obtained in the limit $\sigma_{\|} \rightarrow \infty$. Eliminating the term $\partial_{\| j}$ from Eqs. (71) and (72) and setting $\phi=n$ yields the evolution equation for $\phi$. The set of equations thus obtained provides the fluid model of the ion temperature gradient driven mode or the $\eta_{i}$ mode in sheared magnetic 
fields with finite sheared flows:

$$
\begin{gathered}
\frac{\partial}{\partial t}\left(1-\Delta_{\perp}\right) \phi-\left\{\phi, \Delta_{\perp} \phi\right\}=-\left(1+K \Delta_{\perp}\right) \frac{\partial \phi}{\partial y}-S_{\perp} x\left(1-\Delta_{\perp}\right) \frac{\partial \phi}{\partial y}-S_{\perp} \frac{\partial p}{\partial x \partial y} \\
-\partial_{\|} v+\left\{p, \Delta_{\perp} \phi\right\}+\left\{\frac{\partial p}{\partial x}, \frac{\partial \phi}{\partial x}\right\}+\left\{\frac{\partial p}{\partial y}, \frac{\partial \phi}{\partial y}\right\}-\mu_{\perp}^{(1)} \Delta_{\perp}^{2} \phi \\
\frac{\partial v}{\partial t}+\{\phi, v\}=-S_{\perp} x \frac{\partial v}{\partial y}-S_{\|} \frac{\partial \phi}{\partial y}-\partial_{\|}(p+\phi)+\mu_{\perp}^{(2)} \Delta_{\perp} v+\mu_{\|} \partial_{\|}^{2} v \\
\frac{\partial p}{\partial t}+\{\phi, p\}=-S_{\perp} x \frac{\partial p}{\partial y}-K \frac{\partial \phi}{\partial y}-\Gamma \partial_{\mid} v+\chi_{\perp} \Delta_{\perp} p+\chi_{\|} \partial_{\|}^{2} p .
\end{gathered}
$$

The same domain and the boundary conditions as those for Eqs. (71)-(75) may also be used for Eqs. (78)-(80).

The energy balance equation associated with the set of equations (78)-(80) becomes

$$
\frac{d}{d t} E_{T}=S_{\perp}\left\langle\frac{\partial p}{\partial x} \frac{\partial \phi}{\partial y}\right\rangle-S_{\|}\left\langle v \frac{\partial \phi}{\partial y}\right\rangle-\frac{K}{\Gamma}\left\langle p \frac{\partial \phi}{\partial y}\right\rangle-W_{D}^{0}
$$

where

$$
E_{T^{\prime}}=\frac{1}{2}\left(\left\langle\phi^{2}\right\rangle+\left\langle\left|\nabla_{\perp} \phi\right|^{2}\right\rangle+\left\langle v^{2}\right\rangle+\frac{1}{\Gamma}\left\langle p^{2}\right\rangle\right)
$$

and

$$
\begin{aligned}
W_{D}^{0}= & \mu_{\perp}^{(1)}\left\langle\left|\Delta_{\perp} \phi\right|^{2}\right\rangle+\mu_{\perp}^{(2)}\left\langle\left|\nabla_{\perp} v\right|^{2}\right\rangle+\mu_{\|}^{(2)}\left\langle\left|\partial_{\|} v\right|^{2}\right\rangle \\
& +\frac{\chi_{\perp}}{\Gamma}\left\langle\left|\nabla_{\perp} p\right|^{2}\right\rangle+\chi_{\|} \Gamma\left\langle\left|\partial_{\|} p\right|^{2}\right\rangle .
\end{aligned}
$$

We note that in the limit $\sigma_{\|} \rightarrow \infty$ there is no particle flux $(\langle n \partial \phi / \partial y\rangle=0)$ since we have assumed the adiabatic electron response $(n=\phi)$.

\section{Effects of Electron Temperature Fluctuations}

Since the introduction of of the Hasegawa-Wakatani model ${ }^{10}$ it is common to take the electron temperature as constant. With the neglect of electron temperature fluctuations it is only the 
parallel electrical resistivity that drives the drift wave branch unstable. As shown by Hinton and Horton ${ }^{4}$ and Horton and Varma, ${ }^{5}$ however, in the ${ }_{1}^{\text {s }}$ resence of electron temperature fluctuations the processes of electron parallel diffusivities $\nu_{\| e}$ and $\kappa_{\| e}$ and the thermo-electron effects $\alpha_{\text {thermo }} n_{e} \partial_{\|} T_{e}$ in the electron momentum equation and $\alpha_{\text {thermo }} T_{e} \partial_{\|} j_{l} / e$ in the electron energy balance equation $\left(\alpha_{\text {thermo }}=0.71\right)$ also contribute to the growth rate of the collisional drift wave. In particular, it is known that positive $\eta_{e}\left(=L_{n} / L_{T e}\right)$ can be a strong stabilizing effect on the collisional drift wave. ${ }^{19}$ In this section, we include these effects in the formulation presented in the previous section.

The inclusion of the thermo-electric effect and the electron parallel viscosity generalizes $\mathrm{Eq}$. (2) to

$$
0=-\partial_{\|} n_{e} T_{e}+e n_{e} \partial_{\|} \Phi+e n_{e} \eta j_{\|}-\beta n_{e} \partial_{\|} T_{e}+\frac{4}{3} \nu_{\| e} \partial_{\| i}^{2} v_{\| e}
$$

where the electron parallel viscosity $\nu_{\| e}=\alpha_{\text {visc }} n_{e} T_{e} / \nu_{e}$ with $\alpha_{\text {visc }}=0.4$. The thermal energy balance equation is

$$
\frac{3}{2} n_{e}\left[\frac{\partial}{\partial t}+\left(\mathbf{v}_{E}+\mathbf{v}_{\| e}\right) \cdot \nabla\right] T_{e}+n_{e} T_{e} \partial_{\|} v_{\| e}=\partial_{\|}\left(\kappa_{\| e} \partial_{\|} T_{e}\right)+\frac{\beta}{e} T_{e} \partial_{\|} j_{\|}+\eta j_{\|}^{2}
$$

with the electron parallel thermal diffusivity

$$
\kappa_{\| e}=\alpha_{\text {heat }} \frac{n_{e} T_{e}^{\prime}}{m_{e} \nu_{e}}, \alpha_{\text {heat }}=1.6
$$

where the ratio of specific heats is assumed to be $\gamma=5 / 3$. We note that $n_{e}=n_{i}$ from charge neutrality.

As in Sec. III, we write the electron temperature as $T_{e}=T_{e 0}+\tilde{T}_{e}$ and the electron parallel flow velocity as $v_{\| e}=v_{\| e 0}+\tilde{v}_{\| e}$. For simplicity, the mean flow velocities are taken to be zero $\left(\mathrm{v}_{e 0}=\mathrm{v}_{i 0}=0\right)$ in this section. With the use of dimensionless dependent variables

$$
\begin{aligned}
\mathcal{T}_{e} & =\frac{\tilde{T}_{e}}{T_{0}} \frac{L_{n}}{\rho_{s}}, \quad v_{e}=\frac{\tilde{v}_{\| e}}{c_{s}} \frac{L_{n}}{\rho_{s}}, \\
j & =\frac{L_{n}}{e n_{0} c_{s} \rho_{s}} j_{\|}
\end{aligned}
$$

and dimensionless parameters 


$$
\begin{aligned}
\chi_{\| e} & =\frac{\kappa_{\| e}}{L_{n} c_{s} n_{0}}=\frac{\alpha_{\text {heat }} T_{e 0}}{m_{e} \nu_{e} L_{n} c_{s}}, \\
\mu_{\| e} & =\frac{4 c_{s} \nu_{\| e}}{3 n_{0} T_{e 0} L_{n}}=\frac{4 \nu_{\| e}}{3 m_{i} n_{0} c_{s} L_{n}}, \\
\eta_{e} & =d\left(\ln T_{e 0}\right) /\left(\ln n_{0}\right),
\end{aligned}
$$

Equation (81) can be written as

$$
j / \sigma_{\|}=\tilde{\partial}_{\|}\left(n-\phi+\left(1+\alpha_{\text {thermo }}\right) \mathcal{T}_{e}\right)-\mu_{\| e} \tilde{\partial}_{\|}^{2} v_{e},
$$

where $j$ also satisfies

$$
j=v \cdots v_{e} .
$$

Similarly, the nondimensional form of Eq. (82) becomes

$$
\frac{3}{2}\left(\frac{\partial}{\partial \tilde{t}} \mathcal{T}_{e}+\left\{\phi, \mathcal{T}_{e}\right\}+\eta_{e} \frac{\partial \phi}{\partial \tilde{y}}\right)+\tilde{\partial}_{\|} \nu_{e}=\chi_{\| e} \tilde{\partial}_{\|}^{2} \mathcal{T}_{e}+\alpha_{\text {thermo }} \tilde{\partial}_{\|} j+\frac{1}{\sigma_{\|}} j^{2}
$$

This election thermal balance equation brings in the parameter $\eta_{e}$ from the í $\tilde{E x} d T_{e} / d x$ convection. Replacing Eq. (75) by (83), we obtain the set of equations (71)-(74), (8.3)-(85) (with $S_{.1}=S_{\|}=0$ since the mean flow velocity is assumed to be zero in this section). which governs collisional drift waves under the influence of electron temperature fluctuations.

\section{Linear Dispersion Relatıons}

In this section, we discuss linear aispersion relations of the sysiems derived in Secs. III and IV. Here the local approximation is employed, in which the parallel derivative $\widetilde{\partial}_{\|}$is replaced by a constant $i \tilde{k}_{\|}$. It is also assumed that the mean shear flows are zero $\left(S_{\perp}=S_{\|}=0\right)$ for simplicity. Under these assumptions, the space and time dependence of the normal mode may be given by $\exp i\left(\tilde{k}_{x} \tilde{x}+\tilde{k}_{3}, \tilde{y}+\tilde{k}_{z} \tilde{z}-\tilde{\omega} \tilde{t}\right)$ with $\tilde{k}_{\perp}^{2}=\tilde{k}_{x}^{2}+\tilde{k}_{y}^{2}$ and the constant $\tilde{k}_{\|}$models the operator $\tilde{\partial}_{\|}$with the relations $\tilde{k}_{\|}=s \Delta_{x} \tilde{k}_{y}+\tilde{k}_{z}$, where $\Delta_{x}$ denotes a typical mode width in the $x$-direction. In the case of zero magnetic shear $(s=0)$, the local approximation gives 
the exact dispersion relation of the linearized systems with the relavions $\tilde{k}_{\|}=\tilde{k}_{z}$. With the use of $\Omega=\tilde{\omega} / \tilde{k}_{y}$, the dispersion relation of the system of Eqs. (71)-(75) is given by

$$
\begin{aligned}
\left(1+k_{\perp}^{2}\right) \Omega^{2} & -\left(1-k_{\perp}^{2} K\right) \Omega-\left(\frac{k_{\|}}{k}\right)^{2} \frac{1+\frac{K}{\Omega}}{1-\frac{\Gamma}{\Omega^{2}}\left(\frac{k_{\|}}{k}\right)^{2}} \\
& =i \epsilon_{\eta} \Omega^{2}(\Omega+K)-i\left(\frac{k_{\|}}{k}\right)^{2} \epsilon_{\eta} \frac{K+\Omega}{1-\frac{\Gamma}{\Omega^{2}}\left(\frac{k_{\|}}{k}\right)^{2}},
\end{aligned}
$$

where

$$
\epsilon_{\eta}=\frac{k k_{\perp}^{2}}{\sigma_{\|} k_{\|}^{2}}
$$

$k=\tilde{k}_{y}$ and all the tildes were dropped for simplicity. We also note that all the diffusion coefficients in Eqs. (71)-(75) are ignored for simplicity.

\section{A. Collisionless ion temperature gradient driven modes}

The collisionless limit $\left(\epsilon_{\eta}=0\right)$ of Eq. (86) gives the well-known dispersion relation of the slab-type ion temperature gradient driven mode (Eqs. (67)-(70) with $S_{\perp}=S_{\|}=0$ ). In the presence cl strong ion pressure gradient $(K \gg 1)$, the fastest growing mode is given ${ }^{17}$ by the relation $\tilde{h}_{\perp}^{2}=1 / K(\ll 1)$ with the eigenfrequency

$$
\Omega=\frac{-1+i \sqrt{3}}{2}\left(\left(\frac{\tilde{k}_{\|}}{\tilde{k}_{y}}\right)^{2} K\right)^{1 / 3}
$$

There are also two stable branches with $\tilde{h}_{\perp}^{2}=1 / K$ given by

$$
\Omega=\left(\left(\frac{\tilde{k}_{\|}}{\tilde{k}_{y}}\right)^{2} K\right)^{1 / 3} \text { and } \frac{-1-i \sqrt{3}}{2}\left(\left(\frac{\tilde{k}_{\|}}{\tilde{k}_{y}}\right)^{2} K\right)^{1 / 3} \text {. }
$$

Thus the unstable branch travels in the ion diamagnetic direction and the non-damping stable branch travels in the electron diamagnetic direction. In the dimensionless form, the unstable eigenfrequency may be written in terms of $\omega=\left(c_{s} / L_{n}\right) \Omega \tilde{k}_{y}$ as

$$
\omega=\frac{-1+i \sqrt{3}}{2}\left(-\omega_{p i}^{*} k_{\|}^{2} c_{s}^{2}\right)^{1 / 3}
$$


when

$$
k_{\perp} \rho_{s}=\left(T_{i}\left(1+\eta_{i}\right) / T_{e}\right)^{-1 / 2}
$$

where $\omega_{p i}^{*}=-\left(c T_{i} / e B\right)\left(k_{y} / L_{n}\right)\left(1+\eta_{i}\right)=-\omega_{e}^{*} K, \omega_{e}^{*}=\left(c T_{e} / e B\right) k_{y}, k_{y}=\tilde{k}_{y} / \rho_{s}, \tilde{k}_{\|}=k_{\|} / L_{n}$ and $k_{\perp}=\tilde{k}_{\perp} / \rho_{s}$. This instability results from the coupling of the ion acoustic wave with the thermal mode arising from $\omega_{p i}^{*}{ }^{17}$ More detailed physical interpretation of this mode is given in Sec. VI.

\section{B. Collisional modification of ion temperature gradient driven modes}

The presence of small resistivity $\epsilon_{\eta}$ in Eq. (86) affects the growth rate of the ion temperature gradient driven mode given by Eq. (87). Taking the ordering assumption that $\tilde{k}_{\perp}^{2}=1 / K \ll 1$, $\left(\tilde{k}_{\|} / \tilde{k}_{y}\right)^{2} \ll 1, K / \Omega \gg 1$ and $\epsilon_{\eta} K \ll 1$, we obtain $\Omega=((1+i \sqrt{3}) / 2) K^{1 / 3}\left(\tilde{k}_{\|} / \tilde{k}_{y}\right)^{2 / 3}(1+$ $\left.i \varepsilon_{\eta} K / 3\right)$, or in terms of the dimensional form,

$$
\gamma_{G}=\left(-\omega_{p i}^{*} k_{\|}^{2} c_{s}^{2}\right)^{1 / 3}\left(\frac{\sqrt{3}}{2}-\epsilon_{\eta} \frac{T_{i}}{3 T_{e}}\left(1+\eta_{i}\right)\right)
$$

where

$$
\epsilon_{\eta}=\frac{\omega_{e}^{*} \nu_{e}}{k_{\|}^{2} v_{\mathrm{th}, e}^{2}} k_{\lrcorner}^{2} \rho_{s}^{2}
$$

where $\gamma_{G}$ denotes the growth rate $\gamma_{G}=\operatorname{Im} \omega$ and $v_{\mathrm{th}, e}=\sqrt{T_{e} / m_{e}}$. It is shown that the finite resistivity reduces the growth of the ion temperature gradient driven mode.

\section{Collisional drift wave}

Hasegawa and Wakatani have shown that finite resistivity destabilizes the drift wave. 010 This is a different branch of instability from the instability discussed in the previous subsection and occurs even in the absence of ion temperature gradients. Under the ordering assumption that $\left(\tilde{k}_{\|} / \tilde{k}_{y}\right)^{2} \sim \mathcal{O}\left(\epsilon_{\eta}\right)$ and $\Omega=\Omega_{0}+\Omega_{1}$ with $\left|\Omega_{1} / \Omega_{0}\right| \sim \mathcal{O}\left(\epsilon_{\eta}\right)$, we obtain to the lowest order

$$
\Omega_{0}=\frac{1-\tilde{k}_{\perp}^{2} K}{1+\tilde{k}_{\perp}^{2}} \quad(\sim \mathcal{O}(1))
$$


Here, unlike subsections V.A and V.B, it is assumei that $\tilde{k}_{\perp} \sim K \sim \mathcal{O}(1)$. From Eq. (86), it is easy to obtain the next order expression

$$
\Omega_{1}=\frac{(1+K)}{\left(1-k_{\perp}^{2} K\right)^{2}}\left(\frac{\tilde{k}_{\|}}{\tilde{k}_{y}}\right)^{2}+i \epsilon_{\eta} \frac{(1+K)\left(1-k_{\perp}^{2} K\right)}{\left(1+k_{\perp}^{2}\right)^{3}} .
$$

The growth rate is then given in the dimensional form by

$$
\gamma_{G}=\frac{\omega_{e}^{* 2} \nu_{e}}{k_{\|}^{2} v_{\mathrm{th}, e}^{2}} \frac{k_{\perp}^{2} \rho_{s}^{2}}{\left(1+k_{\perp}^{2} \rho_{s}^{2}\right)^{3}}\left(1+\frac{T_{i}}{T_{e}}\left(1+\eta_{i}\right)\right)\left(1-\frac{T_{i}}{T_{e}}\left(1+\eta_{i}\right) k_{\perp}^{2} \rho_{s}^{2}\right) \text {. }
$$

If the wavelength is long $\left(k_{\perp} \rho_{s} \ll 1\right)$, therefore, finite ion pressure gradients $\left(1+\eta_{i}>0\right)$ are shown to further destabilize the unstable collisional drift wave in Eq. (88).

\section{Effects of electron temperature fluctuations}

Finite electron temperature fluctuations, together with electron diffusivity and thermoelectron effects, alter the growth rate of the collisional drift wave. ${ }^{4,5,20}$ Based on Eqs. (83)-(85) that describe these effects, we examine the growth rate of the collisional drift wave in the subsection. The dispersion relation obtained fiom the system of Eqs. (71)-(74), (83)-(85) is given by

$$
\begin{aligned}
\left(1+k_{\perp}^{2}\right) \Omega^{2} & -\left(1-k_{\perp}^{2} K\right) \Omega-i \epsilon_{\eta} \Omega^{2}(\Omega+K)\left(1+\sigma_{\|} \mu_{\| e} k_{\|}^{2}+\frac{\sigma_{\|}}{\chi_{\| e}} \frac{\left(1+\alpha_{\text {thermo }}\right)^{2}}{\left(1-i \frac{3}{2} \epsilon_{\eta} \frac{\sigma_{\|} \Omega^{2}}{\chi_{\| e} k_{\perp}^{2}}\right)}\right) \\
& +i \epsilon_{\eta} \frac{\sigma_{\|} \Omega^{2}}{\chi_{\| e} k_{\perp}^{2}} \frac{\left(1+\alpha_{\text {thermo }}\right)}{\left(1-i \frac{3}{2} \epsilon_{\eta} \frac{\sigma_{\|} \Omega^{2}}{\chi_{\| e} k_{\perp}^{2}}\right)}\left[\frac{3}{2} \eta_{e}+\left(\frac{k_{\|}}{k}\right)^{2} \frac{K+\Omega\left(1-i \epsilon_{\eta}(K+\Omega)\right)}{\Omega^{2}\left(1-\frac{\Gamma}{\Omega^{2}}\left(\frac{k_{\|}}{k}\right)^{2}\right)}\right] \\
& =\left(1-i \epsilon_{\eta}\left(\frac{k_{\|}}{k_{\perp}}\right)^{2} \Omega \sigma_{\|} \mu_{\| e}\right)\left(\frac{k_{\|}}{k}\right)^{2} \frac{1+K / \Omega-i \epsilon_{\eta}(K+\Omega)}{1-\frac{\Gamma}{\Omega^{2}}\left(\frac{k_{\|}}{k}\right)^{2}},
\end{aligned}
$$

where $k=\tilde{k}_{y}$ and all the tildes were dropped for simplicity. It is easy to see that Eq. (86) is obtained from Eq. (86) in the limit of $\chi_{\| e} \rightarrow \infty$ and $\mu_{\| e} \rightarrow 0$. Here the large $\chi_{\| e}$ limit prevents electron temperature fluctuations from being excited by allowing fast heat transport 
along the magnetic field line while the small $\mu_{i \mid e}$ limit corresponds to the assumption made in deriving Eq. (86) that the electron parallel diffusion $\mu_{\| e}$ is small.

The eigenfrequency $\Omega$ of the collisional drift wave may be obtained from Eq. (89) under the following ordering assumptions: $\epsilon_{\eta}=\tilde{k}_{y} \tilde{k}_{\perp} / \sigma_{\|} \tilde{k}_{\|}^{2} \ll 1, \sigma_{\|} \sim \chi_{\| e}, \sigma_{\|} x_{\| e} \tilde{k}_{\|}^{2} \sim \mathcal{O}(1)$, $\tilde{k}_{\perp}^{2} \sim\left(\tilde{k}_{\|} / \tilde{k}_{y}\right)^{2} \sim \mathcal{O}\left(\epsilon_{\eta}^{1 / 2}\right)$. Writing $\Omega=\Omega_{0}+\Omega_{1}$ with $\left|\Omega_{1} / \Omega_{0}\right| \ll 1$, we obtain the lowestorder frequency $\Omega_{0}$ of the long-wavelength collisional drift wave as $\Omega_{0}=1$. Calculating the higher-order contribution $\Omega_{1}$, we obtain the normalized growth rate

$$
\begin{aligned}
\operatorname{Im} \Omega= & \epsilon_{\eta}\left[(1+K) f_{k_{\|}}-\frac{\sigma_{\|}\left(1+\alpha_{\text {thermo }}\right)}{\chi_{\| e} \tilde{k}_{\perp}^{2}}\left(\frac{3}{2} \eta_{e}+\left(\frac{\tilde{k}_{\|}}{\widetilde{k}^{2}}\right)^{2}(1+K)\right)\right. \\
& \left.-\frac{\tilde{k}_{\|}^{4} \sigma_{\|} \mu_{\| e}}{\widetilde{k}_{\perp}^{2} \tilde{k}_{y}^{2}}(1+K)\right],
\end{aligned}
$$

where

$$
f_{k_{\|}}=1+\tilde{k}_{\|}^{2} \sigma_{\|} \mu_{\| e}+\frac{\sigma_{\|}}{\chi_{\| e}}\left(1+\alpha_{\text {ihermo }}\right)^{2}
$$

In terms of the dimensional form, the growth rate is given by

$$
\begin{aligned}
\gamma_{G} & =\frac{\omega_{e}^{* 2}}{k_{\|}^{2} v_{\mathrm{th}, e}^{2}} \frac{\nu_{e}}{{ }^{2}}\left\{k_{\perp}^{2} \rho_{s}^{2}\left(1+\frac{T_{i}}{T_{e}}\left(1+\eta_{i}\right)\right) f_{k_{\|}}-\left(\frac{1+\alpha_{\text {thermo }}}{\alpha_{\text {heat }}}\right)\left[\frac{3}{2} \eta_{e}+\frac{k_{\|}^{2} c_{s}^{2}}{\omega_{e}^{* 2}}\left(1+\frac{T_{i}}{T_{e}}\left(1+\eta_{i}\right)\right)\right]\right. \\
& \left.-\frac{4}{3} \alpha_{\mathrm{visc}} \frac{v_{\mathrm{th}, e}^{2} k_{\|}^{2}}{\nu_{e}^{2}} \frac{c_{s}^{2} k_{\|}^{2}}{\omega_{e}^{* 2}}\left(1+\frac{T_{i}}{T_{e}}\left(1+\eta_{i}\right)\right)\right\},
\end{aligned}
$$

where

$$
f_{k_{\|}}=1+\frac{4}{3} \alpha_{\mathrm{visc}} \frac{T_{e} k_{\|}^{2}}{m_{e} \nu_{e}^{2}}+\frac{\left(1+\alpha_{\mathrm{the} \text { mo }}\right)^{2}}{\alpha_{\text {heat }}} \text {. }
$$

Here we have used the classical expressions of the parallel electron diffusivities $\kappa_{\| e}=\alpha_{\text {heat }} n_{e} T_{e} / m_{e} \nu_{e}\left(\alpha_{\text {heat }}=1.6\right)$ and $\prime_{\| e}=\alpha_{\text {visc }} n_{e} T_{e} / \nu_{e}\left(\alpha_{\text {visc }}=0.4\right)$ and the thermoelectron effect $\alpha_{\text {thermo }}=0.71$, as given in Sec. IV. We note that the first term in \{\} of the expression of $\gamma_{G}$ in Eq. (90) corresponds to the growth rate of the collisional drift wave (Eq. (88) with $k_{\perp} \rho_{s} \ll 1$ ) enhanced by $f_{k_{\|}}$over the value obtained by only including electrical conductivity $\left(f_{k_{\|}} \rightarrow 1\right)$. It is shown in Eq. (90) that a substantial, positive $\eta_{e}$ can 
be a strong stabilizing effect on the collisional drift wave. The role of the parallel electron viscosity $\alpha_{\text {visc }}$ is more complicated since it contributes both to increasing the enhancement factor $f_{k\|\|}$ and to the direct damping in the last term of the $\gamma_{G}$ formula.

\section{Discussion}

In this paper, we have derived from the electrostatic two-fluid equations the sets of equations governing the nonlinear dynamics of the drift waves in the presence of ion temperature gradients. The derivation is based on the consistent orderings, in which the modes are assumed to be localized on a particular magnetic field line and to fluctuate much faster than the evolution of the mean fields but much more slowly than ion-gyromotion. The effects of mean shear flows and electron temperature fluctuations are also discussed. The final Equations for the collisional drift wave [Eqs. (71)-(75)] and for the collisionless drift wave [Eqs. (78)-(80)] provide the basis for nonlinear analysis of the collisional drift wave instability and the collisionless ion temperature gradient driven instability, respectively.

It is worthwhile to exercise our intuition to draw physical pictures of the instabilities discussed in the previous sections. For this purpose, we consider the simplest possible case, namely, the case of no mean sheared flows $\left(S_{\perp}=S_{\|}=0\right)$, no magnetic shear $(3=0)$ and no diffusion. In order to understand the physical mechanism of the collisionless ion temperature gradient driven mode, however, we need a finite ion pressure gradient $\left(p_{i}(x) \neq\right.$ const. ), which is the free energy source of the mode. On ine other hand, for the collisional drift instability, we consider the effects of a finite density gradient $(n(r) \neq$ const. ), which is the driving force of the drift wave, and finite resistivity, which give rise to the breakdown of the adiabatic electron response $(n \neq \dot{\phi} \phi)$. A finite ion pressure gradient plays a secondary role in the collisional drift instability, modifying its growth rate as shown ir Eq. (88).

Starting with the collisionless ion temperature gradient driven mode, we consider the fastest growing mode whose perpendicular wavenumber $k_{\perp}$ satisfies $k_{\perp}^{2} \simeq K^{-1}$. Suppose 
we have a small positive perturbation of the electrostatic potential $\phi$ in the plasma. This potential perturbation induces a $\mathbf{E} \times \mathbf{B}$ flow circulating around the perturbation, as shown in Fig. 1a. The high pressure ions and low pressure ions are mixed by the $\mathbf{E} \times \mathbf{B}$ flow and this mixture creates a high pressure spot and a low pressure spot on each side of the potential perturbations as shown in Fig. 1b. This convection of the ion pressure is described by Eq. (80) or

$$
\frac{\partial p}{\partial \tilde{t}}=-K \frac{\partial \phi}{\partial \tilde{y}}
$$

where the effect of $\Gamma$ is ignored. The parallel dynamics then plays an important role: into the low pressure spot, plasraas flow from the outside along the magnetic field lines whereas plasmas in the high pressure spot are pushed away along the field lines (Fig. 1c). The left-hand side and the third term of the right-hand side of Eq. (79) or

$$
\frac{\partial v}{\partial \tilde{t}}=-\tilde{\partial}_{\|} p
$$

describes this process. This process, coupled with the effect of nonzero $\Gamma$ (or the parallel compressibility) of Eq. (80), induces the ion acoustic wave, which is destabilized by the ion pressure gradient in this case. Finally, through the balance between the first term of the left-liand side and the parallel compression term of the right-hand side of Eq. (78) or

$$
\frac{\partial \phi}{\partial \tilde{t}}=-\tilde{\partial}_{\|} v
$$

the parallel motion of the plasmas increases the electrostatic potential at the low pressure spot and decreases it at the high pressure spot.

The increased electrostatic potential at the low pressure spot repeats the same sequence of processes and generates more potential perturbations in the negative $\%$-direction or the ion diamagnetic direction. This mechanism thus induces a growing mode travelling in the ion diamagnetic direction (Eq. (87)), which is the ion temperature gradient driven instability. It should be noted that this instability exists even in the absence of the density gradient and 
magnetic shear. On the other hand, the high pressure spot in Fig. 1b, which induces the decrease of the potential, generates a stable branch of the mode travelling in the electron diamagnetic direction.

The collisional drift instability is a higher order correction to the stable drift wave caused by finite resistivity. Ignoring the ion parallel flow and the small contribution from the parallel current $\partial_{\|} j$, we have the linear equation of density fluctuation from Ea. (72)

$$
\frac{\partial n}{\partial \tilde{t}}=-\frac{\partial \phi}{\partial \tilde{y}} .
$$

This is a similar situation described in Figs. $1 \mathrm{a}$ and $\mathrm{b}$, namely, the $\mathbf{E} \times \mathbf{B}$ flow convects the density, instead of the ion pressure. In the case of the adiabatic electrons, the equation above gives the stable drift wave $\Omega=1$ ( or $\omega=\omega_{e}^{*}$ ). However, in the presence of finite resistivity, the electron density fluctuation is related to the potential fluctuation through Eq. (75) or $n=\phi+j /\left(i \tilde{k}_{\|} \sigma_{\|}\right)$. The parallel current $j$ is then related to the polarization current through the relation $\boldsymbol{\nabla} \cdot \mathbf{j}=0(\mathrm{Eq} \cdot(9))$ or

$$
\frac{\partial}{\partial \tilde{t}} \tilde{\Delta}_{\perp} \phi=\tilde{\partial}_{\| j}
$$

which is a simplified form of Eq. (71). Here we note that the right-hand side of Eq. (91) is proportional to the divergence of the polarization current. Therefore the difference between $\tilde{n}$ and $\tilde{\Phi}$ has the $90^{\circ}$ phase shift from $\tilde{\Phi}$ or $n=\phi+i \omega \tilde{k}_{\perp}^{2} \phi / \sigma_{\|} \tilde{k}_{\|}^{2}$, which destabilizes the drift wave. When a finite ion pressure gradient is present $(K \neq 0)$, Eq. $(91)$ is replaced by $(\partial / \partial \tilde{t}-K \partial / \partial \tilde{y}) \tilde{\Delta}_{\perp} \phi=\tilde{\partial}_{\| \mid} j$, as in Eq. (71). This modification by finite $K=T_{i}\left(1+\eta_{i}\right) / T_{e}$ means inclusion of the diamagnetic flow convecting the polarization current. As shown in $\mathrm{Eq}_{\mathrm{l}}$. (88), a finite ion pressure gradient increases the growth rate of the long-wavelength $\left(k_{\perp} \rho_{s} \ll 1\right)$ collisional drift wave instability.

Although the linear analysis based on the local approximation presented in Sec. V reveals generic properties of the instabilities, the eigenvalue analysis is necessary for study of the cross-field mode structure of the instabilities in the presence of the sheared magnetic and 
flow fields. In fact, it has been shown ${ }^{17,18}$ that the strong magnetic shear has a stabilizing effect on the collisionless ion temperature gradient drift instability, which cannot be shown from the local analysis. Our more recent study also shows that finite $\mathbf{E} \times \mathbf{B}$ mean shear flows $\left(S_{\perp} \neq 0\right)$ reduce the growth rate and consequently reduce turbulent transport in the plasma. These stabilizing effects by magnetic and flow shear are of significant importance in practical applications; for example, it is widely believed that improved energy confinement observed in $\mathrm{H}$-mode discharges ${ }^{21}$ are related to reduction of instabilities by the $\mathbf{E} \times \mathbf{B}$ shear flows near the plasma edge. Analysis of such effects by using the linear eigenmode analysis and nonlinear numerical simulations are beyond the goal of the present paper and will be presented elsewhere. 


\section{Appendix A}

In this Appendix we derive Eqs. (38) and (48). It is easy to show that the following identity holds exactly:

$$
\begin{aligned}
& m_{i} n\left(\frac{\partial}{\partial t}+\mathbf{v} \cdot \nabla\right) \frac{\hat{\mathbf{b}} \times \nabla p}{n e B}=-\frac{1}{\omega_{c i} n}(\hat{\mathbf{b}} \times \nabla p)\left(\frac{\partial}{\partial t}+\mathbf{v} \cdot \nabla\right) n \\
& +\frac{1}{\omega_{c i}} \hat{\mathbf{b}} \times \nabla\left(\frac{\partial}{\partial t}+\mathbf{v} \cdot \nabla\right) p+\frac{1}{\omega_{c i}}((\mathbf{v} \cdot \nabla) \hat{\mathbf{b}} \times \nabla p)-\frac{1}{\omega_{c i}}\left(\hat{\mathbf{b}} \times \nabla v_{\alpha}\right) \partial_{\alpha} p
\end{aligned}
$$

where $\mathbf{v}=\left(v_{1}, v_{2}, v_{3}\right)$, the repeated indices are summed from 1 to 3 as before. We also assumed that the unit vector $\hat{b}=\mathbf{B} /|\mathbf{B}|$ is a function of space $\mathbf{x}$ only but $B=|\mathbf{B}|=$ const in time and space, $m_{i}, e$ and $\omega_{c i}=e B / m_{i}$ are also constants, and $n, p$ and $\mathrm{v}$ are functions of times $t$ and space $\mathbf{x}$. The last term of the right-hand side of Eq. (A-1) may be simplified with the use of the following identity, which can be shown to hold exactly after rather tedious calculation

$$
\begin{aligned}
& -2\left(\hat{\mathbf{b}} \times \nabla v_{\alpha}\right) \partial_{\alpha} p+\nabla p \cdot \mathbf{W}^{\mathrm{FLR}}=\left(\partial_{\|} p\right)\left(\hat{\mathbf{b}} \times \nabla_{\perp} v_{\|}-v_{\alpha}\left(\hat{\mathbf{b}} \times \nabla_{\perp}\right) b_{\alpha}+\hat{\mathbf{b}} \times \partial_{\|} \mathbf{v}_{\perp}\right) \\
& +2 \hat{\mathbf{b}}\left(\left(\nabla_{\perp} p \times \hat{\mathbf{b}}\right) \cdot \nabla v_{\|}-v_{\alpha}\left(\left(\nabla_{\perp} p \times \hat{\mathbf{b}}\right) \cdot \nabla\right) b_{\alpha}+\left(\nabla_{\perp} p \times \hat{\mathbf{b}}\right) \partial_{\|} \mathbf{v}\right) \\
& -\hat{\mathbf{b}} \cdot(\nabla \times v) \nabla p+(\nabla \times \mathbf{v}) \partial_{\|} p+\left(\nabla_{\perp} p \times \hat{\mathbf{b}}\right) \nabla_{\perp} \cdot \mathbf{v} .
\end{aligned}
$$

Taking the parallel component of Eq. (A-2) yields

$$
\left(\nabla p \cdot \mathbf{W}^{\mathrm{FLR}}\right)_{\|}=2\left(\left(\nabla_{\perp} p \times \hat{\mathrm{b}}\right) \cdot \nabla v_{\|}-v_{\alpha}\left(\dot{\nabla}_{\perp p} \times \hat{\mathrm{b}}\right) \cdot \nabla b_{\alpha}+\left(\nabla_{\perp} p \times \hat{\mathrm{b}}\right) \partial_{\|} \mathbf{v}\right)
$$

Here the tensor $W^{\mathrm{FLR}}$ is given by Eq. (13), $\widehat{\mathbf{b}}=\left(b_{1}, b_{2}, b_{3}\right), \partial_{\|}=\hat{\mathbf{b}} \cdot \nabla$ and $v_{\|}=\hat{\mathbf{b}} \cdot \mathrm{v}$. It should be noted that in deriving Eqs. (A-1) and (A-2), no assumptions are made on the vector $\mathrm{v}$ and the scalars $p$ and $n$, except that $\mathrm{v}, p$ and $n$ are functions of $t$ and $\mathbf{x}$.

We now assume that $n$ and $\mathrm{v}$ denote the ion density and the ion flow, respectively, and satisfy the continuity equation $\partial n / \partial t+\nabla \cdot(n \mathbf{v})=0(\mathrm{Eq} .(3))$ and $p$ denotes the ion pressure. 
Under the ordering assumptions made in Sec. I-2, or

$$
\begin{array}{ll}
\mathrm{v} / c_{s}=\mathcal{O}(\varepsilon), & \nabla_{\perp} \cdot \mathrm{v}\left(\rho_{s} / c_{s}\right)=\mathcal{O}\left(\varepsilon^{2}\right) \\
p_{0} / n_{i 0} T_{e}=\mathcal{O}(\delta), & \nabla_{\perp} p_{0}\left(\rho_{s} / n_{i 0} T_{e}\right)=\mathcal{O}(\varepsilon) \\
\nabla_{\perp} \tilde{p}\left(\rho_{s} / n_{i 0} T_{e}\right)=\mathcal{O}(\varepsilon), & \nabla_{\perp} v_{\alpha}\left(\rho_{s} / c_{s}\right)=\mathcal{O}(\varepsilon) \\
\partial_{\|} /\left|\nabla_{\perp}\right|=\mathcal{O}(\varepsilon), & \omega_{c i}^{-1} \partial / \partial t=\mathcal{O}(\varepsilon)
\end{array}
$$

the mathematical identities of Eqs. (A-1) and (A-2) may be further simplified. The perpendicular component of Eq. (A-1) becomes

$$
m_{i} n\left(\frac{\partial}{\partial t}+\mathbf{v} \cdot \nabla\right) \mathbf{v}_{d}=\frac{1}{\omega_{c i}} \hat{\mathbf{b}} \times \nabla\left(\frac{\partial}{\partial t}+\mathbf{v} \cdot \nabla\right) p-\frac{1}{\omega_{c i}}\left(\hat{\mathbf{b}} \times \nabla v_{\alpha}\right) \partial_{\alpha} p
$$

up to $\mathcal{O}\left(\varepsilon^{2}\right)$. Here $\mathbf{v}_{d}=(\hat{b} \times \nabla p) / n e B$ and the first term on the right-hand side of Eq. $(A-1)$, which becomes $-(\hat{\mathbf{b}} \times \nabla p)(\nabla \cdot \mathbf{v}) / \omega_{c i} \sim \mathcal{O}\left(\varepsilon^{3}\right)$ with the use of the continuity equation, is dropped. From Eq. (A-2) we obtain

$$
-\frac{1}{\omega_{c i}}\left(\hat{\mathbf{b}} \times \nabla v_{\alpha}\right) \partial_{\alpha} p+\left(\nabla \nu_{i}^{\mathrm{FLR}} \cdot \mathrm{W}^{\mathrm{FLR}}\right)_{\perp}=-\hat{\mathrm{b}} \cdot(\nabla \times \mathrm{v}) \nabla_{\perp} \nu_{i}^{\mathrm{FLR}}
$$

up to $\mathcal{O}\left(\varepsilon^{2}\right)$, where $\nu_{i}^{\mathrm{FLR}}=p / 2 \omega_{c i}$. Combining Eas. (A-4) and (A-5), we obtain

$$
\begin{aligned}
& m_{i} n\left(\frac{\partial}{\partial t}+\mathbf{v} \cdot \nabla\right) \mathbf{v}_{d}+\left(\nabla \cdot \Pi_{i}^{\mathrm{FLR}}\right)_{\perp}=\frac{1}{\omega_{c i}} \hat{\mathbf{b}} \times \nabla\left(\frac{\partial}{\partial t}+\mathbf{v} \cdot \nabla\right) p-\hat{\mathrm{b}} \cdot(\boldsymbol{\nabla} \times \mathbf{v}) \nabla_{\perp} \nu_{i}^{\mathrm{FLR}} \\
& \quad+\nu_{i}^{\mathrm{FLR}}\left(\boldsymbol{\nabla} \cdot \mathbf{W}^{\mathrm{FLR}}\right)_{\perp}+\mathcal{O}\left(\varepsilon^{3}\right)=\frac{1}{\omega_{c i}} \hat{\mathrm{b}} \times \nabla\left(\frac{\partial}{\partial t}+\mathbf{v} \cdot \boldsymbol{\nabla}\right) p+\nabla_{\perp} \zeta+\mathcal{O}\left(\delta \varepsilon^{2}\right), \quad(\mathrm{A}-6)
\end{aligned}
$$

where $\Pi_{i}^{\text {FLR }}=\nu_{i}^{\text {FLR }} \mathbf{W}^{\text {FLR }}, \zeta=-\nu_{i}^{\text {FLR }} \hat{\mathrm{b}} \cdot(\nabla \times \mathrm{v})$ and

$$
\left(\nabla \cdot \mathbf{W}^{\mathrm{FLR}}\right)_{\perp}=-\nabla_{\perp}(\hat{\mathrm{b}} \cdot(\nabla \times \mathbf{v}))+\mathcal{O}\left(\varepsilon^{2}\right)
$$

obtained from Eq. (19) have been used. Equation (A-6) is used to obtain Eq. (48).

The parallel ion momentum equation can also be simplified with the use of Eqs. (13) and $(A-3)$. Under the $\varepsilon$-ordering assumption made in Sec. I-B, it is easy to show that

$$
\left(\hat{\mathrm{b}} \times \nabla_{\perp p}\right) \cdot \nabla v_{\|}+\left(\nabla \frac{p}{2}\right) \cdot \mathbf{W}_{\|}^{\mathrm{FLR}}+\frac{p}{2}\left(\nabla \cdot \mathbf{W}^{\mathrm{FLR}}\right)_{\|}=p \partial_{\|}(\hat{\mathrm{b}} \cdot \nabla \times \mathrm{v})+\mathcal{O}\left(\varepsilon^{3}\right) .
$$

Equation (A-7) is equivalent to Eq. (38). 


\section{Acknowledgments}

The authors wish to thank J.P. Mondt and C.-B. Kirn for helpful discussions. 'This work was supported by the U.S. Department of Energy contract \#DE-FG05-80ET-53088. 


\section{References}

1. L.I. Rudikiov and R.Z. Sagdeev, Dokl. Akad. Nauk SSSR 138, 581 (1961) [Sov. Phys. Dokl. 6, 415 (1961)].

2. B.B. Kadomtsev and O.P. Pogutse, in Reviews of Plasma Physics, edited by M. A. Leontovich (Consultant Bureau, New York, 1965), Vol. 5, p. 249.

3. B. Coppi, M.N. Rosenbluth, and R.Z. Sagdeev, Phys. Fluids 10, 582 (1967).

4. F.L. Hinton and C.W. Horton, Jr., Phys. Fluids 14, 116 (1971).

5. C.W. Horton, Jr. and R.K. Varma, Phys. Fluids 1.5, 620 (1972).

6. S.J. Braginskii, in Reviews of Plasma Physics, edited by M.A. Leontovich (Consultant Bureau, New York, 1965), Vol. I, p. 205.

7. B. Coppi and C. Spight, Phys. Rev. Lett. 41, 551 (1978).

8. T. Antonsen, B. Coppi, and R. Englade, Nucl. Fusion 19, 641 (1979).

9. A. Hasegawa and K. Mima, Phys. Fluids 21, 87 (1978).

10. A. Hasegawa and M. Wakatani, Phys. Rev. Lett. 50, 682 (1983).

11. M. Wakatani and A. Hasegawa, Phys. Fluids 27, 611 (1984).

12. W. Horton, R.D. Estes, and D. Biskamp, Plasma Physics 22, 663 (1980).

13. For example, see W. Horton, Jr., R. Estes, H. Kwak, and D.I. Choi, Phys. Fluids 21, 1366 (1978); P.W. Terry and W. Horton, Phys. Fluids 26, 106 (1983); also see Refs. 14 and 15 .

14. W. Horton, Jr., D.-I. Choi, and W.M. Tang, Phys. Fluids 24, 1077 (1981). 
15. D. Brock and W. Horton, Plasma Physics 24, 271 (1982).

16. For example, see D.L. Brower, W.A. Peebles, S.K. Kim, N.C. Luhmann, W.M. Tang, and P.E. Phillips, Phys. Rev. Lett. 59, 48 (1987); F.X. Sölder, E.R. Müller, F. Wagner, H.J.S. Bosch, A. Eberhagen, H.U. Fahrbach, G. Fussmann, O. Gehre, K. Gentle, J. Gernhardt, O. Gruber, W. Herrmann, G. Janeschitz, M. Kornherr, K. Krieger, H.M. Mayer, K. McCormick, H.D. Murmann, J. Neuhauser, R. Nolte, W. Poschenrieder, H. Röhr, K.-H. Steuer, U. Stroth, N. Tsois, and H. Verbeek, Prys. Rev. Lett. 61, 1105 (1988); R.J. Fonk, R. Howell, K. Jaehnig, L. Roquemore, G. Schilling, S. Scott, M.C. Zarnstorff, C. Bush, R. Goldston, H. Hsuan, D. Johnson, A. Ramsey, J. Schivell, and H. Towner, Phys. Rev. Lett. 63, 520 (1989).

17. S. Hamaguchi and W. Horton, Phys. Fluids B 2, 1833, (1990).

18. S. Hamaguchi and W. Horton, "Ion Temperature Gradient Driven Turbulence in the Weak Density Gradient Limit." IFSR\#430, to appear in Phys. Fluids B.

19. G.W. Hammett and F.W. Perkins, Phys. Rev. Lett. 64, 301 (1990).

20. W. Horton, "Nonlinear Drift Waves and Transport in Magnetized Plasma," IFSR\#416, to appear in Physics Reports.

21. F. Wagner, G. Becker, K. Behringer, D. Campbell, A. Eberhagen, W. Engelhardt, G. Fussmann, O. Gehre, J. Gernhardt, G.v. Gierke, G. Haas, M. Huang, F. Karger, M. Keilhacker, O. Klüber, M. Kornherr, K. Lackner, G. Lisitano, G.G. Lister, H.M. Mayer, D. Meisel, E.R. Müller, H. Murmann, H. Niedermeyer, W. Poschenrieder, H. Rapp, H. Röhr, F. Schreider, G. Siller, E. Speth, A. Stäbler, K.H. Steuer, G. Venus, O. Vollmer, and Z. Yü, Phys. Rev. Lett. 49, 1408 (1982). 


\section{Figure Captions}

Figure Ia $\mathrm{A}$ small positive perturbation of the electrostatic potential $\Phi$ causing a $\mathbf{E} \times \mathbf{B}$ flow.

Figure $\mathrm{Ib}$ The $\mathrm{E} \times \mathrm{B}$ flow viewed from the $z$-direction.

Figure Ic High and low $p_{i}$ spots created by the $\mathrm{E} \times \mathrm{B}$ flow. White arrows indicate plasma flows along the magnetic field lines. 


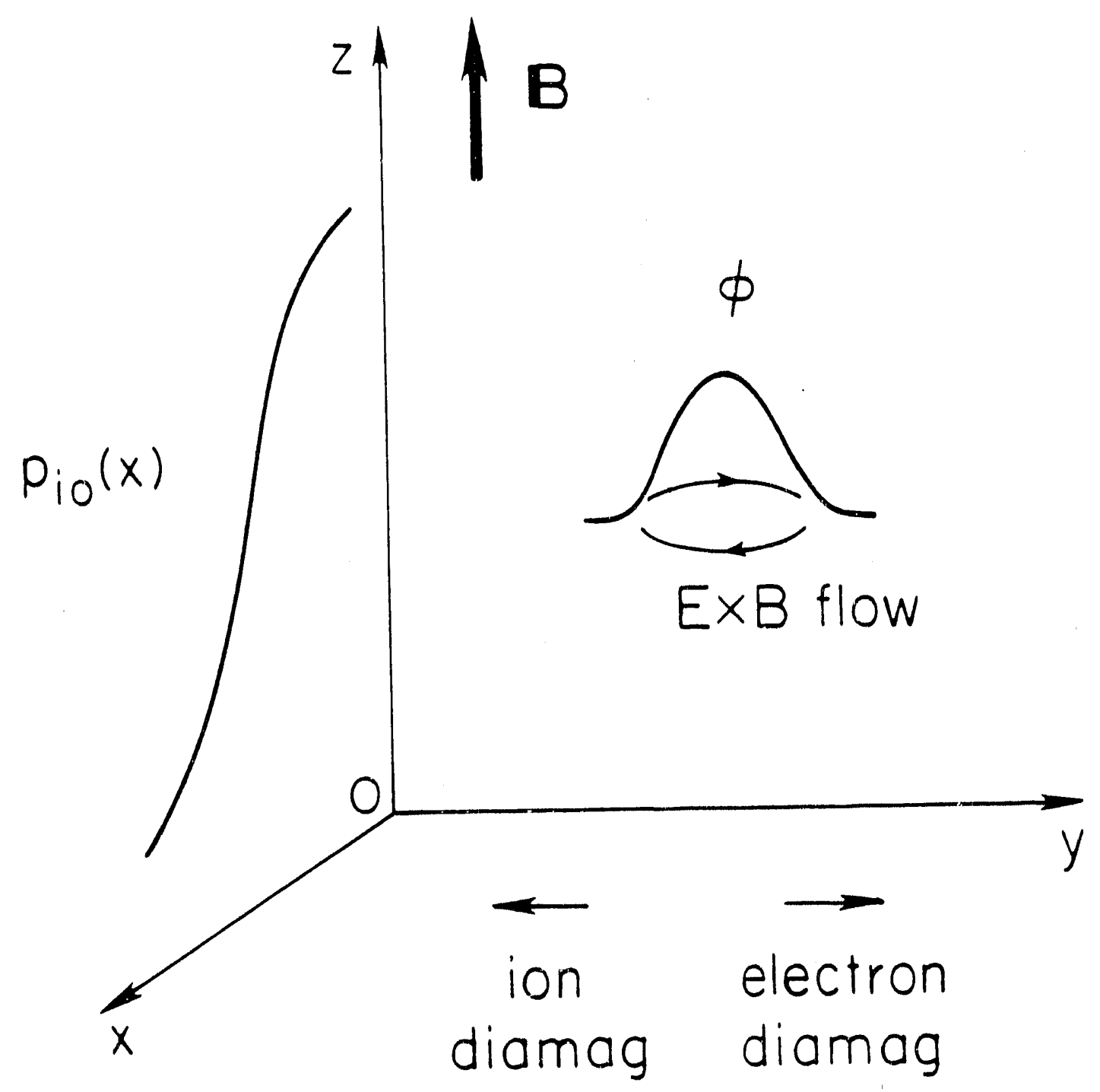

Fig. 1a 


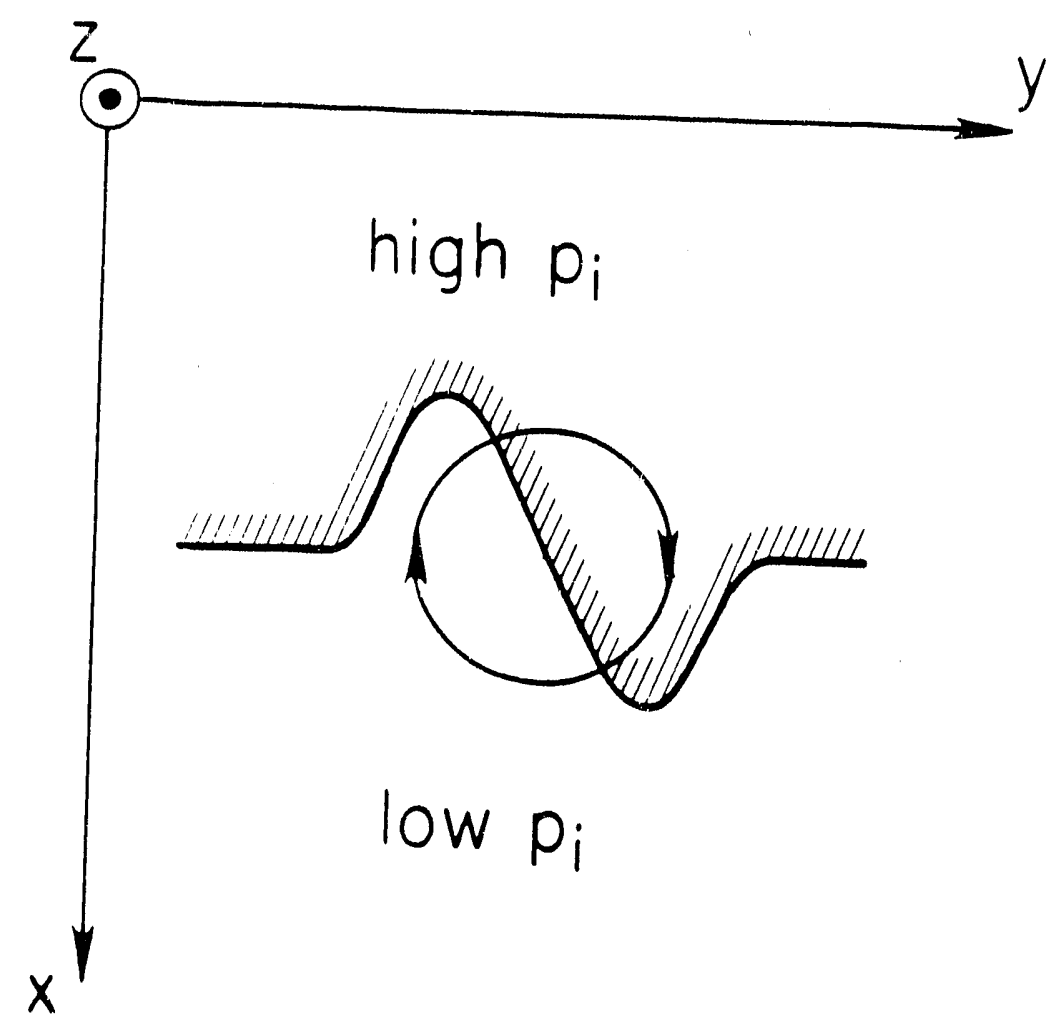

Fig. 1b 


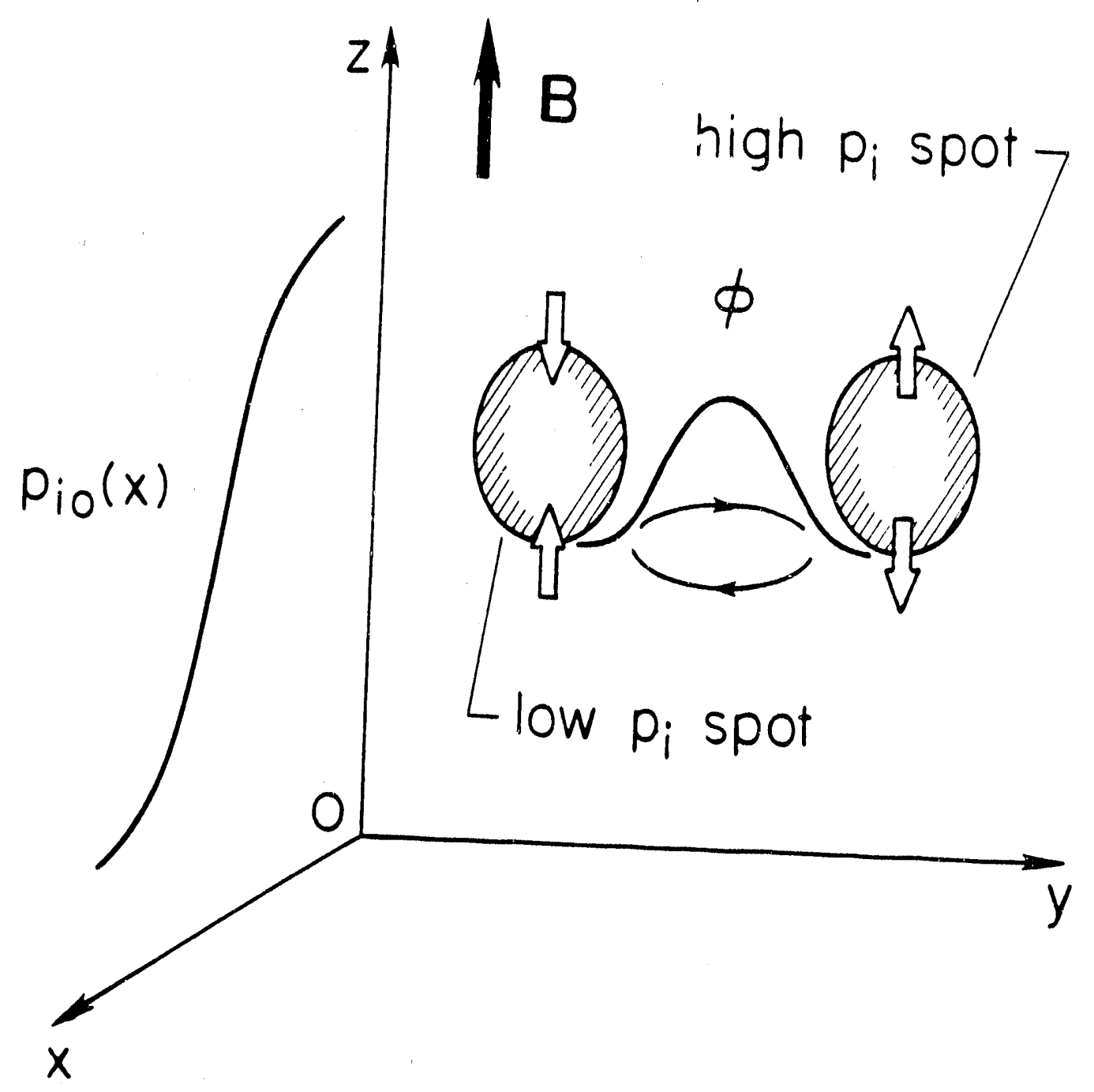

Fig. 1c 

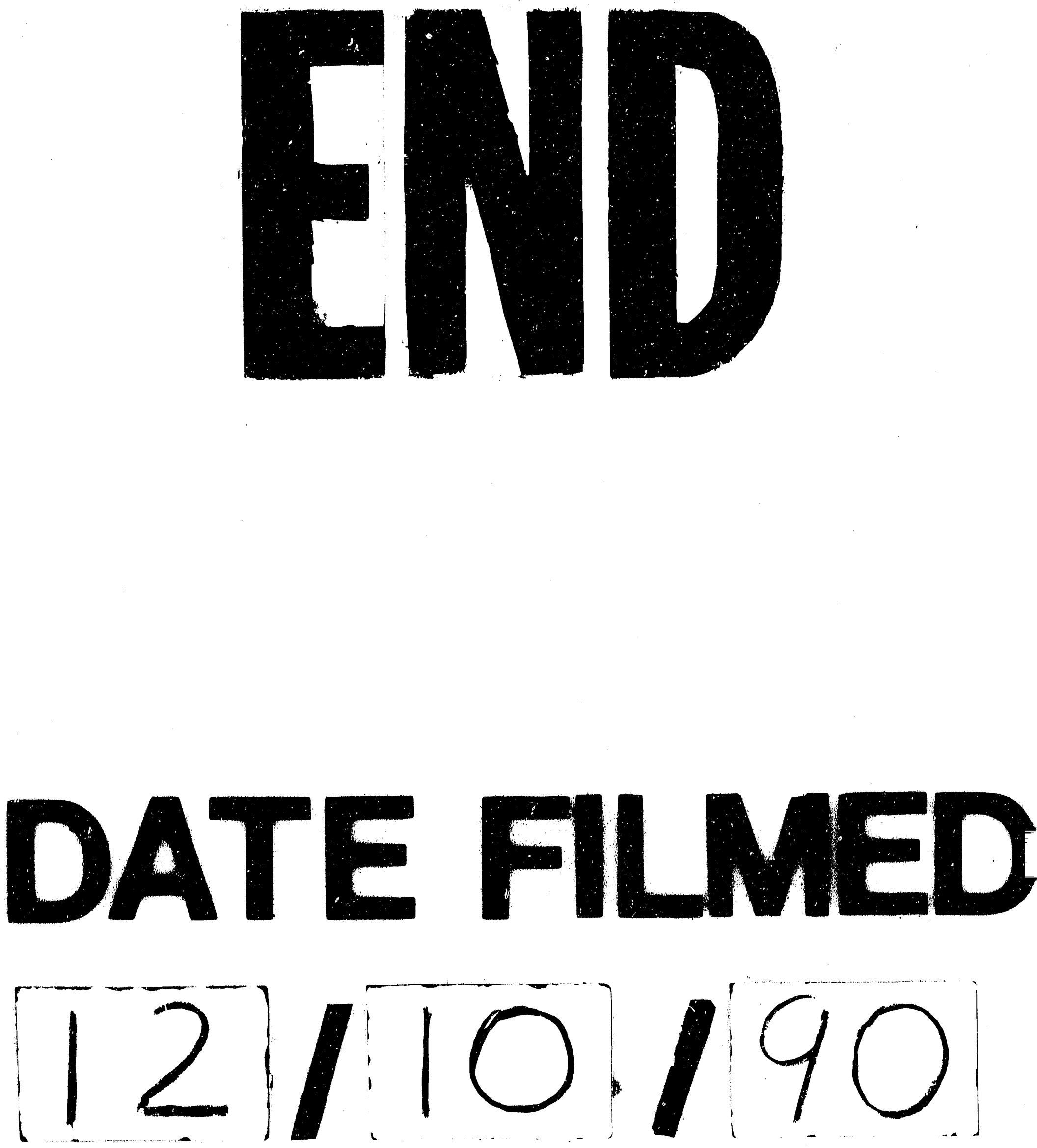
